\title{
Transcriptome Profiling of Toxoplasma gondii-Infected Human Cerebromicrovascular Endothelial Cell Response to Treatment with Monensin
}

\author{
Mohammad S. R. Harun ${ }^{1}\left(\mathbb{D}\right.$, Mica Taylor ${ }^{2}$, Xing-Quan Zhu ${ }^{3, * \mathbb{C}}$ and Hany M. Elsheikha ${ }^{2, *(\mathbb{D})}$ \\ 1 Infectomics Cluster, Advanced Medical \& Dental Institute, Universiti Sains Malaysia, Bertam, Kepala Batas, \\ Pulau Pinang 13200, Malaysia; mosyamsulre@usm.my \\ 2 Faculty of Medicine and Health Sciences, School of Veterinary Medicine and Science, University of \\ Nottingham, Sutton Bonington Campus, Loughborough LE12 5RD, UK; micataylor@hotmail.co.uk \\ 3 State Key Laboratory of Veterinary Etiological Biology, Key Laboratory of Veterinary Parasitology of Gansu \\ Province, Lanzhou Veterinary Research Institute, Chinese Academy of Agricultural Sciences, \\ Lanzhou 730046, China \\ * Correspondence: xingquanzhu1@hotmail.com (X.-Q.Z.); hany.elsheikha@nottingham.ac.uk (H.M.E.); \\ Tel.: +86-(0)931-834-2837 (X.-Q.Z.); +44-(0)115-951-6445 (H.M.E); Fax: +44-(0)115-951-6440 (H.M.E.)
}

Received: 2 May 2020; Accepted: 1 June 2020; Published: 4 June 2020

\begin{abstract}
Central to the progression of cerebral toxoplasmosis is the interaction of Toxoplasma gondii with the blood-brain barrier (BBB) endothelial cells. In the present work, we tested the hypothesis that inhibition of Wnt pathway signalling by the monovalent ionophore monensin reduces the growth of $T$. gondii infecting human brain microvascular endothelial cells (hBMECs) or microglial cells. The anti-parasitic effect of monensin (a Wnt signalling inhibitor) on the in vitro growth of T. gondii tachyzoites was investigated using two methods (Sulforhodamine B staining and microscopic parasite counting). The monensin inhibited T. gondii growth (50\% inhibitory concentration $\left.\left[\mathrm{IC}_{50}\right]=0.61 \mu \mathrm{M}\right)$ with a selective index $=8.48$ when tested against hBMECs $(50 \%$ cytotoxic concentration $\left.\left[\mathrm{CC}_{50}\right]=5.17 \mu \mathrm{M}\right)$. However, $\mathrm{IC}_{50}$ of monensin was $4.13 \mu \mathrm{M}$ with a $\mathrm{SI}=13.82$ when tested against microglia cells $\left(C_{50}=57.08 \mu \mathrm{M}\right)$, suggesting less sensitivity of microglia cells to monensin treatment. The effect of $T$. gondii on the integrity of the BBB was assessed by the transendothelial electrical resistance (TEER) assay using an in vitro human BBB model. The results showed that $T$. gondii infection significantly decreased hBMECs' TEER resistance, which was rescued when cells were treated with $0.1 \mu \mathrm{M}$ monensin, probably due to the anti-parasitic activity of monensin. We also investigated the host-targeted effects of $0.1 \mu \mathrm{M}$ monensin on global gene expression in hBMECs with or without $T$. gondii infection. Treatment of hBMECs with monensin did not significantly influence the expression of genes involved in the Wnt signalling pathway, suggesting that although inhibition of the Wnt signalling pathway did not play a significant role in T. gondii infection of hBMECs, monensin was still effective in limiting the growth of $T$. gondii. On the contrary, monensin treatment downregulated pathways related to steroids, cholesterol and protein biosynthesis and their transport between endoplasmic reticulum and Golgi apparatus, and deregulated pathways related to cell cycle and DNA synthesis and repair mechanisms. These results provide new insight into the host-modulatory effect of monensin during $T$. gondii infection, which merits further investigation.
\end{abstract}

Keywords: Toxoplasma gondii; monensin; TEER; gene expression; Wnt signaling

\section{Introduction}

Toxoplasma gondii is an obligate intracellular apicomplexan protozoan parasite that is widely distributed throughout the world. The disease associated with T. gondii infection, toxoplasmosis, 
can lead to severe central nervous system (CNS) pathologies [1]. Although T. gondii infection is usually asymptomatic in healthy individuals, infection can be fatal in immunocompromised patients and in congenitally infected fetuses [2,3]. Given the pathological damage and neuroinflammation that can be caused by T. gondii infection of the brain [4,5], many epidemiological studies have shown an association between T. gondii infection and a number of neuropsychiatric conditions, such as schizophrenia and bipolar disorder [4-6]. Besides the lack of a vaccine, current treatment options for toxoplasmosis are limited and responses to commonly used medicines are often unsatisfactory [7]. There is a clear need for new and more potent anti-T. gondii therapeutics to reduce the clinical impact of toxoplasmosis. Ideal therapeutic agents should prevent parasite growth, without perturbing the proliferation or homeostasis of host cells. However, in general, although available therapeutic agents display potent anti-T. gondii properties, many are cytotoxic to mammalian cells [8].

The blood-brain barrier (BBB) is the initial site of interaction between neuro-pathogens such as T. gondii and the mammalian brain [9]. Breaching this protective biophysical barrier is a key mechanism by which T. gondii tachyzoites invade and damage the CNS [9]. During T. gondii infection, different signalling pathways control the expression of a wide range of genes that orchestrate molecular and cellular events to eliminate the invading parasite and regulate the associated inflammation [10]. One of these pathways is the Wnt pathway, which is considered an important regulatory axis in the immune system, where genes involved in this pathway were upregulated in T. gondii-infected mice [10]. Moreover, other intracellular pathogens have been shown to manipulate this pathway to facilitate their entry into the cell and establish infection, such as Chlamydia trachomatis [11] and the influenza virus [12].

Small molecule inhibitors of the Wnt pathway are becoming more prominent in the pharmaceutical industry [13], and are increasingly being used to target cancer cells and intracellular pathogens [14]. Natural polyether ionophores, such as monensin, have been substances of great interest, because of their antimicrobial activities [15]. Monensin has been extensively used in veterinary applications as a growth promoter [16] and to treat coccidiosis and cryptosporidiosis [15,17]. In addition, monensin was proposed as an anticancer agent $[18,19]$. Monensin, secreted by the bacteria Streptomyces cinnamonensis, has been shown to modulate Wnt signalling by antagonizing $\beta$-catenin and the lipoprotein receptor-related protein LRP [18]. Investigation into how manipulation of the Wnt pathway by monensin can influence the growth of $T$. gondii could be beneficial in the development of new anti-T. gondii therapeutics.

In this study, we investigated whether inhibition of the Wnt signalling pathway by monensin can reduce the growth of $T$. gondii infecting human brain microvascular endothelial cells (hBMECs) or microglial cells, and whether suppression of the growth of T. gondii within hBMECs using monensin can restore the impairment of the BBB integrity. We also performed systems-level transcriptional analysis of hBMECs infected by $T$. gondii in the presence or absence of monensin to obtain a comprehensive insight into how monensin treatment alters gene expression of hBMECs during T. gondii infection.

\section{Materials and Methods}

\subsection{Chemicals}

Monensin was purchased as a sodium salt powder from Alfa Aesar (Ward Hill, MA, USA) and $1.73 \mathrm{mg}$ was dissolved in $250 \mu \mathrm{L}$ of absolute methanol. The $10 \mathrm{mM}$ of stock solution was diluted with complete culture medium to prepare five working concentrations $0.01 \mu \mathrm{M}, 0.1 \mu \mathrm{M}, 1 \mu \mathrm{M}, 10 \mu \mathrm{M}$ and $100 \mu \mathrm{M}$, which were stored at $4{ }^{\circ} \mathrm{C}$ until use. Sulforhodamine B (SRB) was purchased from

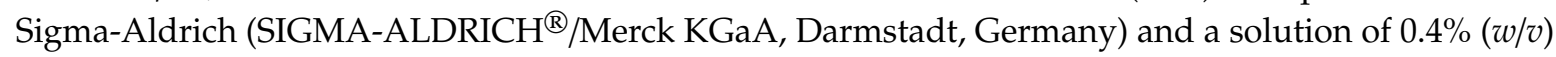
SRB dissolved in $1 \%$ acetic acid (Fisher Scientific, Leicestershire, UK) was prepared and stored protected from light at $4{ }^{\circ} \mathrm{C}$. 


\subsection{Cell Lines}

Primary human brain microvascular endothelial cells (hBMECs) kindly provided by Naveed Khan (American University of Sharjah, Sharjah, UAE) were maintained in modified Gibco ${ }^{\circledR}$ Roswell Park Memorial Institute (RPMI) 1640 media (Thermo Fisher Scientific, Waltham, MA, USA) containing 20\% Gibco ${ }^{\circledR}$ Heat Inactivated Fetal Bovine Serum (HI-FBS), 1\% Gibco ${ }^{\circledR}$ non-essential amino-acids $(100 \times)$, $1 \%$ Gibco $^{\circledR}$ sodium pyruvate $(100 \mathrm{mM}), 1 \%$ Gibco ${ }^{\circledR}$ MEM vitamins $(100 \times), 1 \%$ L-glutamine $(200 \mathrm{mM})$, and $1 \%$ Gibco $^{\circledR}$ antibiotic-antimycotic solution $100 \times(10,000$ units $/ \mathrm{mL}$ of penicillin, $10,000 \mu \mathrm{g} / \mathrm{mL}$ of streptomycin, and $25 \mu \mathrm{g} / \mathrm{mL}$ of Amphotericin B). Human microglial cells (ATCC CRL-3304) originally obtained from the American Type Culture Collection were grown in modified Gibco ${ }^{\circledR}$ Dulbecco's Modified Eagle Medium (DMEM; Thermo Fisher Scientific, Waltham, MA, USA) containing $5 \%$ Gibco ${ }^{\circledR}$ HI-FBS and 1\% Gibco ${ }^{\circledR}$ antibiotic-antimycotic solution 100X. Both cell lines were passaged twice a week and grown in T75 $\left(75 \mathrm{~cm}^{2}\right)$ NUNC ${ }^{\text {TM }}$ tissue culture flasks (Fisher Scientific, Leicestershire, UK) for two weeks before being used in the experiments. All cultures were maintained in a cell culture incubator at $37^{\circ} \mathrm{C}$ with $5 \%$ atmospheric $\mathrm{CO}_{2}$.

\subsection{Parasite Strain and Culture Conditions}

Tachyzoites of the T. gondii RH strain were cultured in NUNC ${ }^{\text {TM }}$ T75 tissue culture flasks containing hBMECs using the same cell culture conditions described above. The tachyzoites were harvested from their feeder cell cultures when $70-80 \%$ of the hBMECs were lysed in about three days. Then, they were separated from host cell debris via centrifugation at $500 \mathrm{~g}$ for $5 \mathrm{~min}$. Based on the cell line used in the subsequent experiments, the number of tachyzoites was adjusted with the respective medium (RPMI for hBMECs or DMEM for microglial cells) to achieve a multiplicity of infection (MOI) of 5 (5 tachyzoites: 1 cell) for each cell line.

\subsection{Efficacy of Monensin on the In Vitro Growth of T. gondii}

We used parasite counting assay in 24-well plates and the sulforhodamine B (SRB) assay in 96-well plates to investigate the effects of inhibition of the Wnt signalling pathway using monensin on the intracellular growth of T. gondii.

\subsubsection{Parasite Counting Assay}

Microglial and hBMEC cultures were seeded on Nunclon ${ }^{\mathrm{TM}}$ Delta Surface 24-well culture plates (Thermo Fisher Scientific, Waltham, MA, USA) at seeding densities of $5 \times 10^{4}$ cells and $10^{5}$ cells per well, respectively. Cultures of both cell types were infected with $T$. gondii tachyzoites at a MOI of 5 . Approximately $3 \mathrm{~h}$ post-infection, the culture medium in each well was discarded and replaced with $1 \mathrm{~mL}$ of fresh medium supplemented with one of the five working concentrations of monensin $(0.01 \mu \mathrm{M}$, $0.1 \mu \mathrm{M}, 1 \mu \mathrm{M}, 10 \mu \mathrm{M}$ and $100 \mu \mathrm{M})$. Growth of cell cultures was monitored daily by microscopic observation using a Zeiss Axiovert 25 inverted microscope (Leica Microsystems, Milton Keynes, UK) with a 10X objective lens. When the parasites had completed a few cycles of development and began to exit the cells in large numbers at $\sim 3$ days after infection of the untreated cells, the absolute number of tachyzoites in each well of treated and untreated cultures was counted. To achieve this, the remaining medium in the wells was topped up with phosphate buffered saline (PBS) with pipetting to produce a homogenous parasite suspension. A small amount $(50 \mu \mathrm{L})$ of the suspension from each well was used to count tachyzoites using a standard hemocytometer counting slide.

\subsubsection{Colorimetric SRB Assay}

The SRB assay involves the measurement of cellular protein content using a plate reader to quantify the absorbance after cells have been fixed and stained with SRB dye. The SRB absorbance values represent the spectrophotometric quantification of protein concentration of the cells, which is directly proportional to the number of cells (i.e. increased absorbance correlates with increased protein 
content, which reflects an increase in the cells' number). Hence, SRB absorbance values can be used as an indicator of host cell proliferation rates in T. gondii-infected versus mock-infected (control) cultures to determine the level of T. gondii growth indirectly. Based on this rationale, we can expect infected cells to produce less absorbance due to the disruption of cell proliferation caused by the damaging effects of the parasite growth on the host cell viability. Here, a microplate-based SRB staining assay was used as a simple, cost-effective, convenient approach to determine the inhibitory effect of monensin on T. gondii growth in vitro. Briefly, cells were cultured in Corning ${ }^{\circledR}$ Costar ${ }^{\circledR}$ 96-well plates (Sigma-Aldrich/Merck KGaA, Darmstadt, Germany). Each well was seeded with $5 \times 10^{3}$ microglial cells or $10^{4} \mathrm{hBMECs}$ in $100 \mu \mathrm{L}$ of the respective medium and then grown to confluency ( 1 day). Tachyzoites used to infect each well at a MOI of 5 were able to invade the host cells within $3 \mathrm{~h}$. Then, the medium was discarded to remove any extracellular tachyzoites and $100 \mu \mathrm{L}$ of media containing one of the five concentrations of monensin $(0.01 \mu \mathrm{M}, 0.1 \mu \mathrm{M}, 1 \mu \mathrm{M}, 10 \mu \mathrm{M}$, and $100 \mu \mathrm{M})$ was added to each well. Wells that received medium without monensin (untreated) served as controls. After five days of incubation, the SRB assay was performed according to Vichai and Kirtikara [20]. Ice-cold $100 \mu \mathrm{M}$ of $10 \%$ trichloroacetic acid (TCA) solution (Fisher Scientific, Leicestershire, UK) was added to each well. The plates were kept at $4{ }^{\circ} \mathrm{C}$ for $1 \mathrm{~h}$. Afterwards, the solution was discarded from the wells followed by thorough washing with distilled water. Next, $25 \mu \mathrm{L}$ of SRB dye was added to each well and incubated for $15 \mathrm{~min}$ at ambient temperature. The plates were wrapped with foil to protect the dye from the light. Then, the wells were washed with $1 \%$ acetic acid to remove any unattached stain, followed by addition of $100 \mu \mathrm{L}$ of $10 \mathrm{mM}$ Tris base solution (Sigma-Aldrich/Merck KGaA, Darmstadt, Germany) to each well. Finally, the plates were gently agitated on a plate shaker at 35 rotations/min for $5 \mathrm{~min}$ to ensure a homogeneous solution. The absorbance was measured at $492 \mathrm{~nm}$ using a plate reader (LT-4000 Microplate Reader, LabTech International Ltd., Heathfield, UK).

\subsection{Effect of Monensin on BBB Integrity}

The hBMECs are fundamental constituents of the BBB and play important roles in the maintenance of barrier integrity. To determine if and to what extent integrity of hBMECs is affected by exposure to $0.1 \mu \mathrm{M}$ monensin in the presence or absence of T. gondii infection, transendothelial electrical resistance (TEER) was measured. Briefly, $0.5 \mu \mathrm{M}$ PET-inserts (24-well Millicell ${ }^{\circledR}$ Cell Culture Inserts, Millipore, Watford, UK) were fitted to wells of the 24-well culture plates. Then, $10^{4}$ cells in $100 \mu \mathrm{L}$ of RPMI medium were added to each insert and $100 \mu \mathrm{L}$ of the culture medium was added to the bottom of the well. The plate was incubated for $1 \mathrm{~h}$; then the medium was discarded and replaced with $1.3 \mathrm{~mL}$ fresh media in the bottom of each well. Also, $300 \mu \mathrm{L}$ fresh media was added onto the cell monolayer inside the inserts. Three days after seeding the cells, the media in the inserts and wells were discarded and replaced with fresh media. Six days after seeding, one of the plates was infected with $T$. gondii by removing old media and adding tachyzoites at a MOI of 5, in fresh medium, to the inserts. The plate was incubated for $1 \mathrm{~h}$ before addition of monensin-supplemented RPMI or fresh RPMI (control) to hBMEC monolayer growing on the apical (top) side of insert. TEER was measured in each insert daily for 4 successive days. TEER was measured using an Evom voltohmmeter with the Endohm 12 and STX2 electrodes (World Precision Instruments, Inc., Sarasota, FL, USA). The electrodes were placed into the insert membrane and bottom of each well. TEER values were measured three times from each well, while changing the position of the electrodes slightly each time in order to obtain the overall average of the insert resistance. A background resistance was established by placing the electrodes into inserts with a blank medium only, without cells. This value was used to calculate the TEER value of each well using the equation (TEER (cells) $=($ TEER(total) - TEER(blank) ) culture area). The culture area of the insert was $0.33 \mathrm{~cm}^{2}$. The TEER value was expected to increase with increased resistance of the hBMEC monolayer. 


\subsection{Microarray Analysis}

The aim of this experiment was to identify the effect of $0.1 \mu \mathrm{M}$ monensin on the overall expression of host cell genes, and in particular on the expression of genes involved in the Wnt signalling pathway. We used cDNA microarrays to detect changes in mRNA expression in four experimental groups. These included: uninfected, un-treated hBMECs (Control); hBMECs treated with $0.1 \mu \mathrm{M}$ monensin for $24 \mathrm{~h}$ (Monensin_only); T. gondii infected-hBMECs for $24 \mathrm{~h}$ (TG_only); and T. gondii-infected hBMECs treated with $0.1 \mu \mathrm{M}$ monensin after $3 \mathrm{~h}$ of infection and incubated for further $24 \mathrm{~h}$ (TG_Monensin).

Total RNA was isolated using RNeasy Mini Kit (Qiagen, Manchester, UK) and eluted with nuclease-free water. The extracted RNA was stored at $-80{ }^{\circ} \mathrm{C}$ for one week prior to microarray analysis. All subsequent sample handling, labelling, and GeneChip (GeneChip Human Gene 1.0 ST arrays, Affymetrix, Santa Clara, CA, USA) processing were performed at the transcriptomics service at Nottingham Arabidopsis Stock Centre (NASC). The RNA concentration and quality were assessed using the Agilent 2100 Bioanalyzer (Agilent Technologies Inc., Palo Alto, CA, USA) and the RNA 6000 Nano kit (Caliper Life Sciences, Mountain View, CA, USA), respectively, prior to the microarray analysis. Only RNA samples with a minimum RNA concentration of $100 \mathrm{ng} / \mu \mathrm{L}$ and RNA integrity number (RIN) value $>8$ were used for microarray analysis.

Single-stranded complimentary DNA was prepared from $200 \mathrm{ng}$ of total RNA as per the Ambion ${ }^{\circledR}$ WT Expression Kit's instructions (Thermo Fisher Scientific, Paisley, UK) for Affymetrix GeneChip Whole Transcript WT Expression Arrays (Affymetrix, Wycombe, UK). Total RNA was first converted to cDNA, followed by transcription to make cDNA. Single-stranded cDNA was synthesized, end labelled, and hybridized for $16 \mathrm{~h}$ at $45^{\circ} \mathrm{C}$ to Human Gene $1.0 \mathrm{ST}$ arrays (Affymetrix, Wycombe, UK). All liquid handling steps were performed by a GeneChip Fluidics Station 450 and GeneChips were scanned with the GeneChip Scanner 3000 7G (Affymetrix, Wycombe, UK) using Command Console v3.2.4. The microarray data have been deposited in the ArrayExpress database at EMBL-EBI (Available online: www.ebi.ac.uk/arrayexpress) under accession number E-MTAB-8817.

The R package software (R Studio Inc., Boston, MA, USA) was used to process the data. Briefly, a data file, called 'pdatamon' was created to link each microarray data file with '.ga.cel' ending to one of the four experimental groups (i.e. Control, Monensin_only, TG_only, TG_Monensin). The libraries of modules (oligo, gplots, RColorBrewer, pvclust, colourspace, limma, gene filter, hugene21sttranscriptcluster.db) needed for quality control, differential gene expression and transcript cluster ID annotation were downloaded and uploaded into the workspace. Next, Affymetrix microarray data files were uploaded together with 'pdatamon' file. Quality control analysis and normalization were performed using the robust multi-array average (RMA). RMA normalization is sensitive for low expression values, and provides consistent fold-change estimates, and excellent background adjustment compared to MAS 5.0 and dChip normalization methods [21,22]. Only genes exhibiting a fold-change FC $\leq-1$ (downregulated) or FC $\geq 1$ (upregulated) relative to control and an adjusted $p$-value $<0.05$ were considered as differentially expressed genes (DEGs). The ranked list of DEGs were included in subsequent analyses. Comparison was made between control (untreated + uninfected) hBMECs and each of the other three groups, including hBMECs treated with monensin (Monensin_only), T. gondii-infected hBMECs treated with monensin (TG_Monensin), and hBMECs infected with T. gondii only (TG_only).

\subsection{Gene Ontology (GO) Analysis}

Functional annotations of the valid DEGs and statistical overrepresentation of the GO terms in the categories of biological processes, molecular functions, and cell components [23], were retrieved in accordance with instructions [24,25], using the PANTHER online database (Available online: www.pantherdb.org). The PANTHER library of annotated genes (Homo sapiens) was used as reference list for calculating statistical overrepresentation with a false discovery rate (FDR) $<0.05$ considered significantly enriched. 


\subsection{Pathway Enrichment Analysis}

The DEGs genes were functionally annotated and positioned within the respective biological pathways using the Reactome pathway database (Available online: https://reactome.org) and the Reactome Analysis Tool version v65 (Available online: https://reactome.org/PathwayBrowser/\#TOOL= AT). Significance was estimated using hypergeometric testing and FDR was controlled for using the Benjamini-Hochberg procedure. Pathways with FDR $<0.01$ were considered significantly enriched.

\subsection{Statistical Analysis}

Statistical analysis was performed using a Student's $t$-test (two independent groups), one-way ANOVA (multiple groups, one independent factor) or two-way ANOVA (multiple groups, two independent factors) followed by a Bonferroni-Holm post hoc test using GraphPad Prism 7 software for Windows (GraphPad Software, San Diego, CA, USA). The mean of at least three independent experiments is presented, with error bars in the graphs showing the standard error of the mean (SEM). Differences were considered statistically significant at a $p$ value of $<0.05$. The level of significance was reported as: ${ }^{*} p<0.05,{ }^{* *} p<0.01,{ }^{* * *} p<0.001$, and ${ }^{* * *} p<0.0001$. The drug concentration that caused $50 \%$ inhibition of host cell growth was expressed as $50 \%$ cytotoxic concentration $\left(\mathrm{CC}_{50}\right)$. The $\mathrm{CC}_{50}$ of monensin on microglia and hBMECs were calculated by plotting dose-response curves followed by performing simple linear regression analysis using Graph Pad Prism 7 software. The calculation of the half-maximal inhibitory concentration $\left(\mathrm{IC}_{50}\right)$, which is the concentration of monensin that caused a $50 \%$ decrease of T. gondii growth compared to the control in both cell lines, were calculated using Graph Pad Prism 7's nonlinear regression (curve fit) built-in analysis tool of dose-response inhibition (three parameters). The selectivity index (SI), which represents the ratio of the $\mathrm{CC}_{50}$ for host cells to the $\mathrm{IC}_{50}$ for T. gondii, was calculated by comparing the cytotoxicity of monensin for host cells to that of T. gondii.

\section{Results}

\subsection{Antiparasitic Effect of Monensin}

The tachyzoite counts were carried out to measure the effect of monensin on the in vitro growth of T. gondii. The results showed a significant decrease $(p<0.01$; ANOVA) in the number of counted tachyzoites in a dose-dependent manner, and $>90 \%$ and $70 \%$ inhibition of $T$. gondii was achieved at $1 \mu \mathrm{M}$ monensin concentration in hBMECs and microglial cells, respectively. The inhibitory effect of monensin on parasite growth was relatively greater in infected hBMEC cultures from $0.1 \mu \mathrm{M}$ to $100 \mu \mathrm{M}$ concentration. However, $0.1 \mu \mathrm{M}$ was considered the minimal concentration to cause significant antiparasitic effects because no significant reduction in the tachyzoite counts was caused by higher concentrations and the reduction was not significantly different between hBMECs and microglia cells (Figure 1).

It was important to test the effects of monensin on hBMECs and microglia in the absence of T. gondii infection. The SRB-based results showed lack of significant difference in cell density levels between infected and non-infected cultures of both hBMECs and microglia at low levels of monensin, suggesting that the number of treated + infected cells did not significantly decrease more than the control cells, probably due to inhibition of T. gondii infection by monensin (Figure 2). We performed $t$-tests to draw comparisons on the effect of each concentration of monensin between infected and non-infected cultures of each cell line. None of the data showed any significant differences except the $10 \mu \mathrm{M}$ treated-hBMEC cultures and $100 \mu \mathrm{M}$ treated-microglia cultures. The density of both infected and non-infected hBMEC cultures showed significant decrease $(p<0.05)$ within the entire range of the tested concentrations $(0.01 \mu \mathrm{M}$ to $100 \mu \mathrm{M})$. However, when the same SRB assay was performed on microglia culture (infected and non-infected), there was no significance reduction in cell density. 


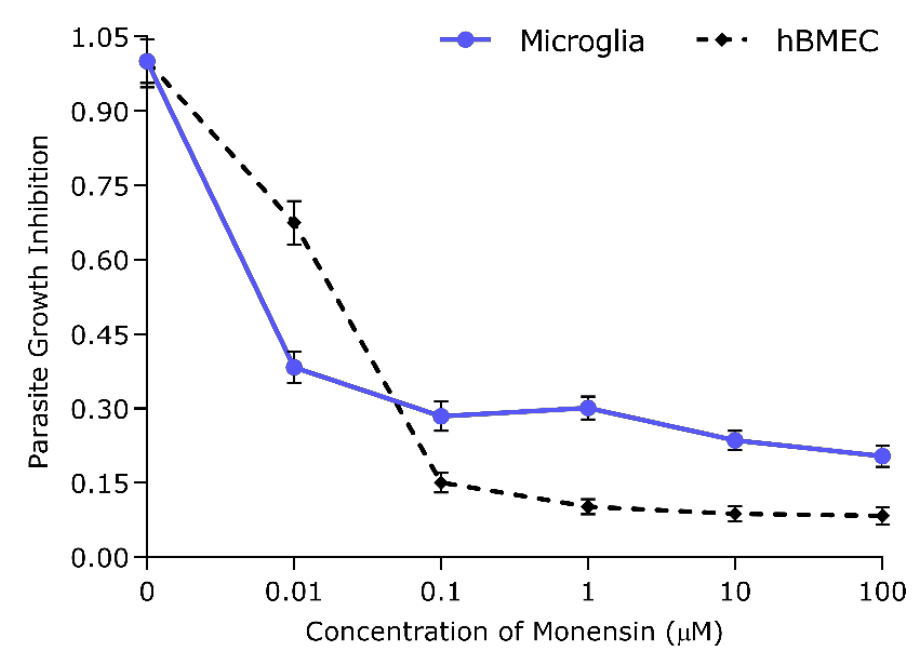

Figure 1. Toxoplasma gondii tachyzoite growth inhibition in microglia and human brain microvascular endothelial cell (hBMEC) cultures was dependent on monensin concentration. The parasite growth was inhibited (compared to parasite growth in untreated cells) as the concentration of monensin increased, but at $0.1 \mu \mathrm{M}$ the inhibitory effect reached a plateau. Data represent mean values $( \pm \mathrm{SEM})$ of triplicate from two independent experiments for each cell line.

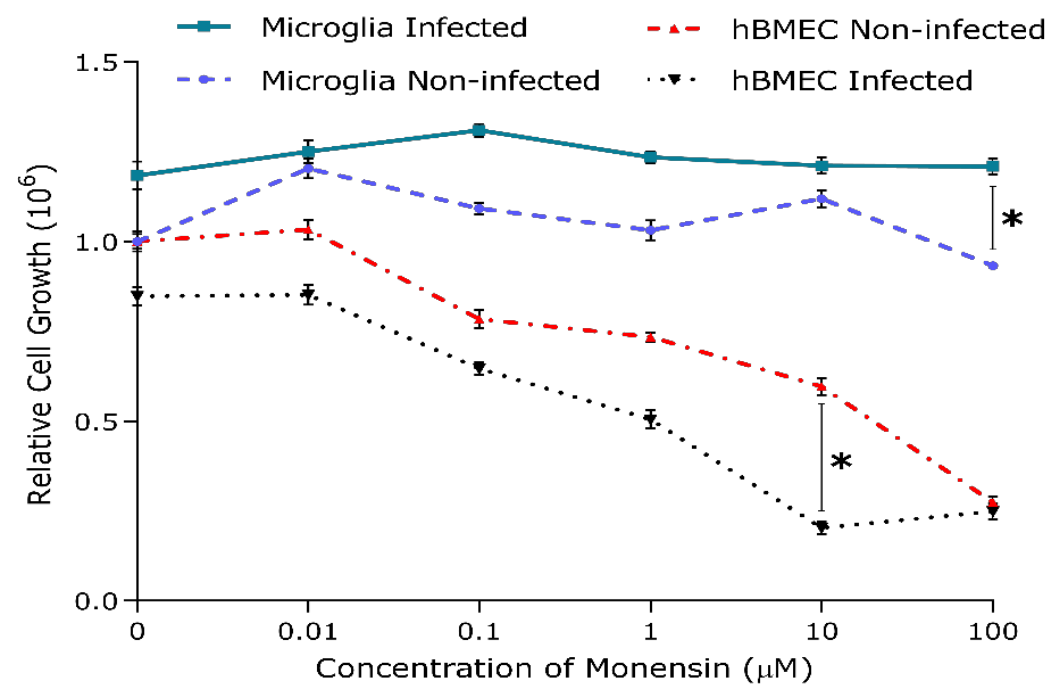

Figure 2. Microglia and hBMEC cultures showed different sensitivity to monensin. Relative growth of each cell line was assessed by the sulforhodamine B (SRB) assay. The relative absorbance values of infected and treated cells were compared to non-infected and treated (control) cells. A significant decrease in cell number was observed in infected and uninfected hBMECs with increasing monensin concentration compared to microglia cultures. Data represent mean values ( $\pm \mathrm{SEM}$ ) of five replicates from three independent experiments for each cell line. Multiple Student $t$-tests; ${ }^{*}, p<0.05$.

It is worthwhile to highlight that the SRB approach does not distinguish between anti-T. gondii effect and host cell toxicity characteristics, since death of host cells carrying the parasite could be related to cell toxicity caused by monensin as well as, or in lieu of, any anti-T. gondii effect. Therefore, it is possible that the anti-T. gondii effect of monensin in infected hBMECs may be confounded by the toxic effect of monensin on these cells. The $50 \%$ inhibitory concentration $\left(\mathrm{IC}_{50}\right)$ for $\mathrm{T}$. gondii tachyzoites $(0.61 \mu \mathrm{M})$ and half-maximal cytotoxic concentrations $\left(\mathrm{CC}_{50}\right)$ for hBMECs was $5.17 \mu \mathrm{M}$. The selective index (SI) was calculated as the $\mathrm{CC}_{50} / \mathrm{IC}_{50}$ of monensin and was found to be 8.48. However, the observed effects of monensin seem to be specific to host cell sensitivity to monensin and independent of the direct anti-T. gondii effect, since treatment with monensin did not induce the same cytotoxic effect in microglia cultures, where $\mathrm{IC}_{50}$ of monensin was $4.13 \mu \mathrm{M}$ with a $\mathrm{SI}=13.82$ when tested against 
microglia $\left(\mathrm{CC}_{50}=57.08 \mu \mathrm{M}\right)$. These results show that although monensin reduced the growth rates of T. gondii tachyzoites in both hBMECs and microglia cells, the SI was higher in microglia cells compared to hBMECs, suggesting less sensitivity of microglia cells to monensin treatment.

\subsection{Assessment of hBMEC Monolayer Integrity}

We investigated how hBMEC integrity was affected by T. gondii infection and/or modulated by Wnt inhibition via exposure to $0.1 \mu \mathrm{M}$ monensin. The effect of monensin on the TEER of infected and uninfected hBMECs was monitored over 4 days. At one day post infection, there was no significant ( $p \geq 0.05$; $t$-tests) difference between $T$. gondii-infected cells treated with $0.1 \mu \mathrm{M}$ monensin compared to infected + untreated, probably because it takes about a day for infection to establish. However, from day 2 after infection there was a significant decrease in hBMECs' TEER between $0.1 \mu \mathrm{M}$ monensin-treated and untreated infected cells. Interestingly, infected and treated cells did not show any significant difference throughout the entire experiment, suggesting that cell integrity was maintained by monensin treatment, possibly via inhibiting the damage caused by the parasite growth (Figure 3). On the contrary, the infected and untreated cells showed a significant reduction in TEER readings compared to the infected and treated cells $(p<0.05)$, suggesting that $T$. gondii infection significantly compromised host cell integrity. There was a $38.2 \%$ decrease in the resistance of infected + untreated cells compared to infected + treated cells relative to the control cells. Interestingly, hBMECs' resistance did not change significantly from day to day and cell integrity was generally stable over the time period for control, infected + treated, and $0.1 \mu \mathrm{M}$ monensin only groups. These findings are in line with the results of the anti-T. gondii effect of monensin (Figures 1 and 2), suggesting that monensin had offset the cellular damage through inhibition of the parasite growth within the hBMECs and, hence, enhanced the recovery of cell integrity, leading to better endothelial resistance than infected + untreated cells.

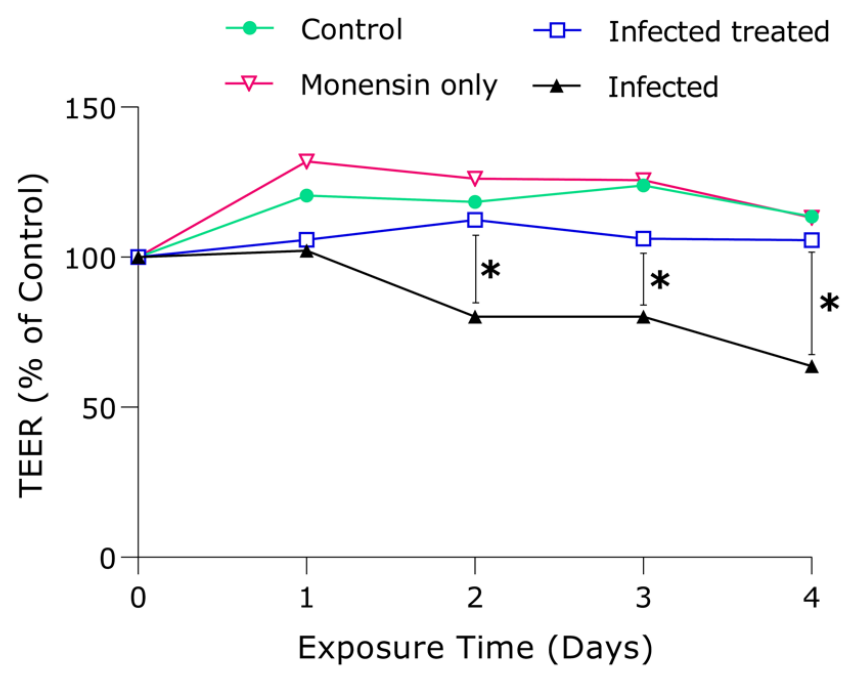

Figure 3. The effect of $0.1 \mu \mathrm{M}$ monensin on the transendothelial electrical resistance (TEER) of hBMEC monolayer. Cells grown on the membrane of transwell inserts, a simplified blood-brain barrier model, were treated with $0.1 \mu \mathrm{M}$ monensin or medium only (control) in the presence or absence of T. gondii infection. Infected and monensin-treated monolayer maintained their TEER value over four days, but, in non-treated and infected monolayer, a significant reduction in TEER values was observed (Multiple Student $t$-tests; $\left.{ }^{*}, p<0.05\right)$. The data shown represent the means of six replicates from two separate experiments and the error bars indicate the S.E.M.

\subsection{Microarray Quality Control Analysis}

The transcriptome analysis included four experimental groups: (i) uninfected and untreated hBMECs (Control-C2YA; C2YB; C2YC); (ii) uninfected hBMECs treated for $24 \mathrm{~h}$ with $0.1 \mu \mathrm{M}$

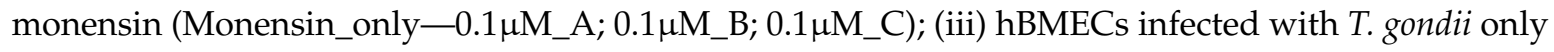


(TG_only-Inf 24 A; Inf 24 B; Inf 24 C); and (iv) T. gondii-infected hBMECs treated for $24 \mathrm{~h}$ with $0.1 \mu \mathrm{M}$ monensin after $3 \mathrm{~h}$ of infection (TG_Monensin-TG $+0.1 \mu \mathrm{MA}$; TG $+0.1 \mu \mathrm{MB} ; \mathrm{TG}+0.1 \mu \mathrm{MC}$ ). The $0.1 \mu \mathrm{M}$ concentration was selected for the array experiment because preliminary data revealed that this concentration was just sufficient to trigger the cell's transcriptional response (unpublished data) and also to avoid the growth inhibition of hBMECs that would have occurred at higher concentrations. The overall distribution between microarray files of the examined samples indicated that normalization was required before the samples are analyzed. As shown in the pre-normalization box plot (Figure S1A), the distributions of all sample median expression values were not uniform before normalization, which might lead to under- or over-estimated expression values if samples were compared. Following normalization, the median expression values of all samples became more uniform (Figure S1B).

\subsection{Correlation Between Samples}

Hierarchical clustering was performed to show the level of correlation between the transcriptional profiles of all 12 samples (based on the top 50 most significantly expressed genes) and identified four distinct subgroups (Figure 4A). Based on the dendrogram, four experimental groups, representing 12 expression profiles, were segregated into four clusters. These four clusters corresponded to four groups (TG_only, TG_Monensin, Monensin_only, Control_only) where three replicates within each group had similar expression patterns. As shown in the dendrogram, the expression profile of TG_only group was different from the expression profiles of the other three groups, namely Control_only, Monensin_only and TG_Monensin. Principal component analysis (PCA) also showed similar clustering of samples into four distinct groups, where samples within each group were clustered together. However, despite the high degree of concordance between the replicates per group, the TG $+0.1 \mu \mathrm{M} \_\mathrm{C}$ sample did not fully cluster with the other two replicates of the TG_Monensin group, which might be attributed to sample to sample variation within the group (Figure 4B). Moreover, TG_Monensin group and Monensin_only group samples clustered together and their gene expression profiles were more similar than those of the TG_only group samples. The overall expression values between the three experimental groups compared to the Control_only group is displayed as a Venn diagram (Figure 5). This diagram shows the shared and unique DEGs between the three comparison groups. Table S1 lists 19 DEGs that are shared by all three comparison groups. The TG_only had more total DEGs compared to the other two groups (Monensin_only and TG_Monensin).

\subsection{Expression Patterns of Altered Genes}

Here, the transcriptional response of hBMECs treated with monensin in the presence or absence of T. gondii infection was investigated in order to obtain more insight into the mechanism of action of monensin against T. gondii.

\subsubsection{Control vs. Monensin_only}

Treatment of hBMECs with $0.1 \mu \mathrm{M}$ monensin induced more upregulation of genes than downregulation (Figure 6A). A total of 480 DEGs were identified; 368 were upregulated and 112 were downregulated. Among the 480 DEGs, 244 were not annotated (NA) and 236 were annotated. Out of the annotated genes, 171 were upregulated and 65 were downregulated. The top 30 most significant DEGs induced by monensin treatment and their name, gene symbol, gene description, and $\log _{2}$ FC values are listed in Table S2. The most significantly annotated gene was 'DNA damage inducible transcript $3^{\prime}$ (DDIT3) with a $\log _{2}$ FC of 2.984. In addition, the top 30 upregulated and downregulated genes are listed in Tables S3 and S4, respectively. Among these, the most highly upregulated gene was also DDIT3, whereas the most highly downregulated gene with annotation was 'small nucleolar RNA, C/D box 116-24' (SNORD116-24) with a $\log _{2}$ FC of -2.543 . The heatmap shows a correlation between samples based on the top 30 most significant DEGs after $24 \mathrm{~h}$ treatment with $0.1 \mu \mathrm{M}$ monensin (Figure 6D). 
A

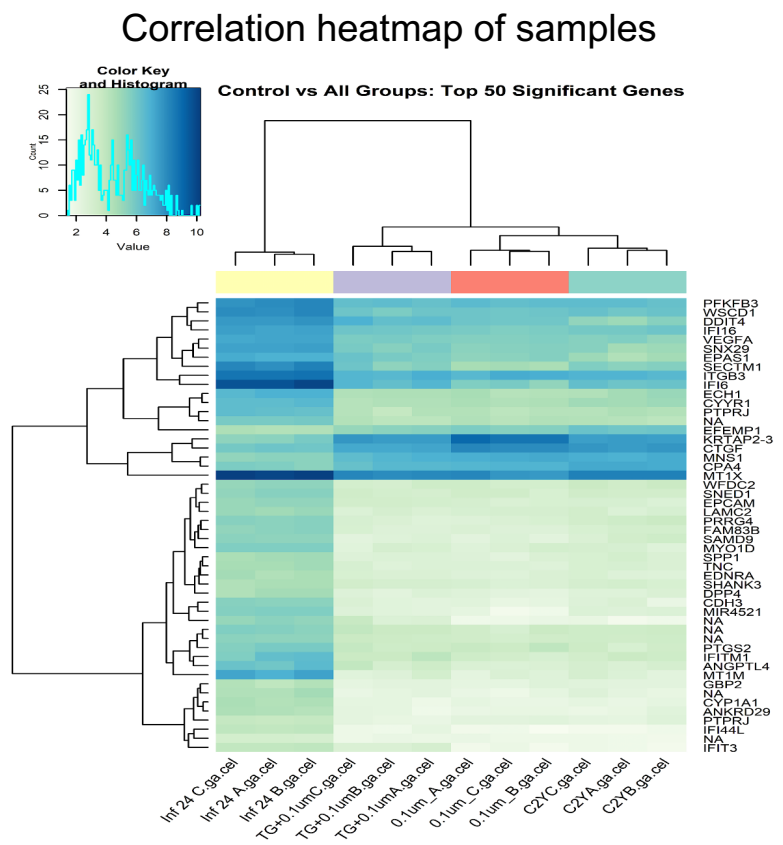

B

Principal Component Analysis

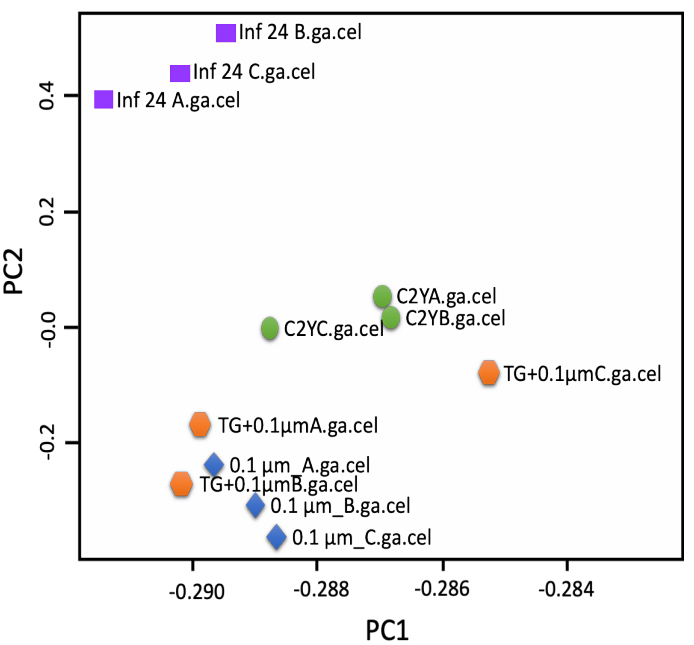

Figure 4. (A) A heatmap showing the correlation between all 12 microarray transcriptome expression profiles based on the top 50 most significantly expressed genes. Shades of light green and dark blue represent low and high correlation, respectively. Based on the dendrogram, four groups of the 12 sample expression profiles were clustered into four distinct clusters, where three samples within each group had similar expression profiles. (B) Principal component analysis (PCA) plot also shows that samples within each group were similar to each other, with the only exception being the dataset of TG_Monensin group samples.

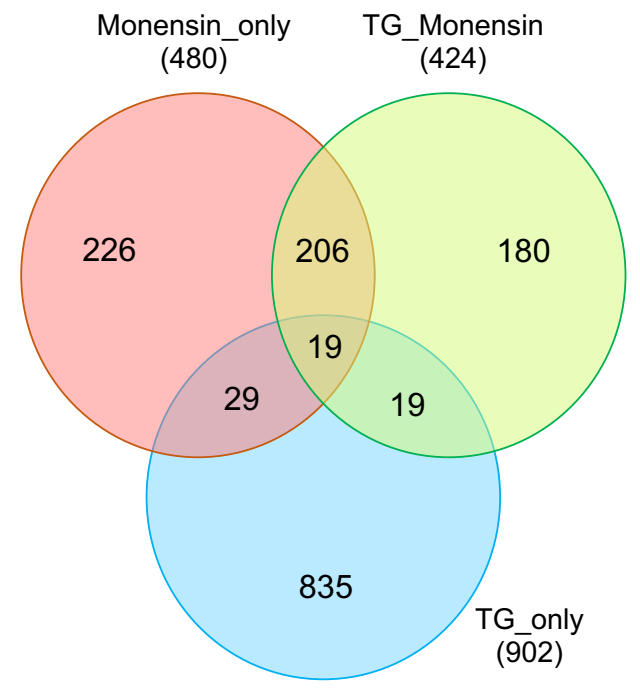

Figure 5. Three-way Venn diagram comparing expression profiles of Control vs. TG_only, Control vs. Monensin_only and Control vs. TG_Monensin. Numbers in the overlapped areas (i.e. at the intersection of the circles) represent DEGs shared between groups. The shaded area in the middle represents 19 DEGs shared between the three groups (Table S1).

\subsubsection{Control vs. TG_Monensin}

The overall expression profile of hBMECs infected with T. gondii and treated with $0.1 \mu \mathrm{M}$ monensin (TG_Monensin) for $24 \mathrm{~h}$ is shown (Figure 6B). Overall, there were more upregulated genes 
than downregulated genes. A total of 424 DEGs passed the selection criteria, of these 134 were upregulated and 290 were downregulated. Among the 424 DEGs, 114 were without annotation (NA) while 310 were annotated. Focusing on the annotated genes, 201 of them were upregulated, but 109 were downregulated. The top 30 most significant DEGs in hBMECs infected with T. gondii and treated with $0.1 \mu \mathrm{M}$ monensin (TG_Monensin) are listed in Table S5. Among these, the most highly significant upregulated genes with annotation were DDIT3 with a $\log _{2}$ FC of 2.446 and 'homocysteine inducible ER protein with ubiquitin-like domain 1' (HERPUD1) with a $\log _{2}$ FC of 2.199. The top 30 upregulated and downregulated genes are listed in Tables S6 and S7, respectively. The most upregulated gene was 'uncharacterized LOC105369371' (LOC105369371) with a $\log _{2}$ FC of 5.135 and the most downregulated gene was 'endothelin 1 ' (EDN1) with a $\log _{2}$ FC of -1.960 . Figure $6 \mathrm{E}$ depicts the heatmap showing a correlation between samples based on to the top 30 most significant DEGs in hBMECs infected with T. gondii and treated with $0.1 \mu \mathrm{M}$ monensin (TG_Monensin).

A

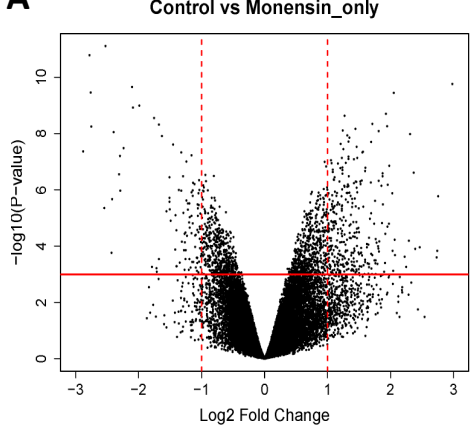

D

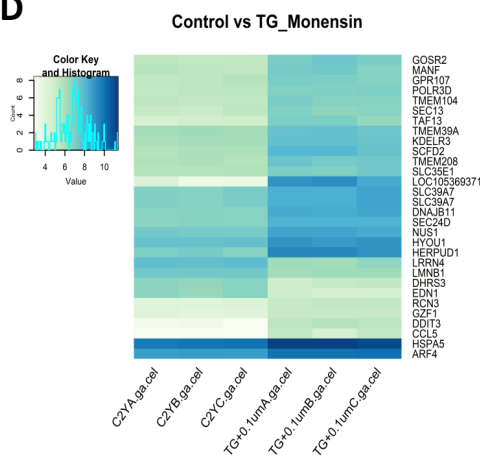

B

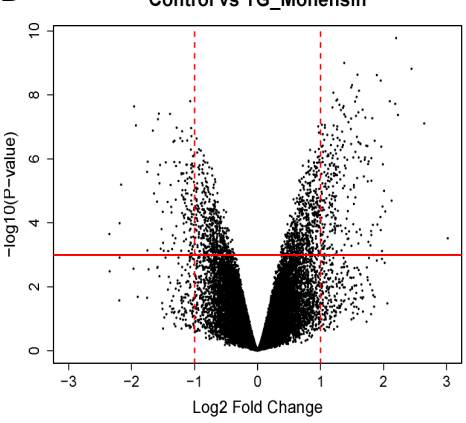

E

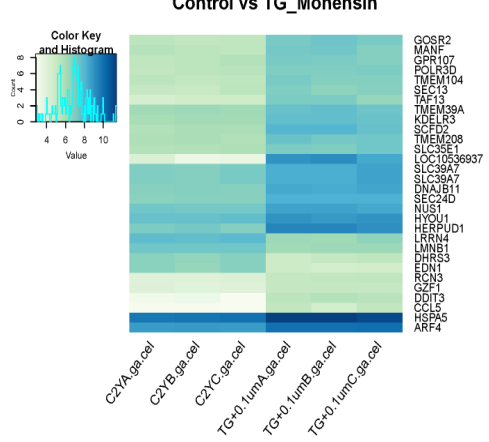

C

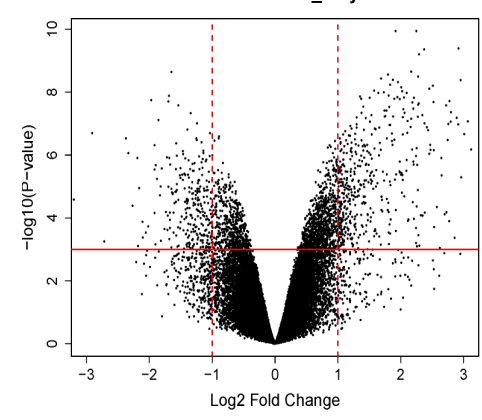

$\mathbf{F}$

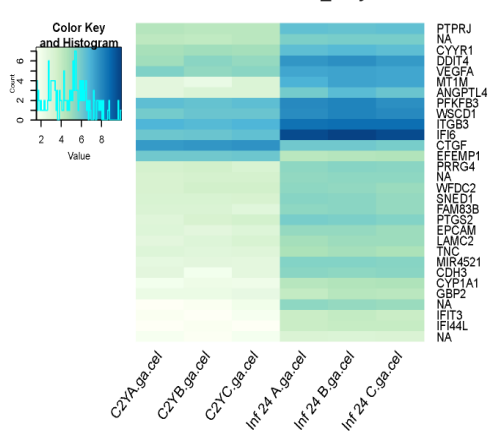

Figure 6. (A-C) Volcano plots showing the expression profile of hBMECs treated with $0.1 \mu \mathrm{M}$ monensin (Monensin_only), hBMECs treated with $0.1 \mu \mathrm{M}$ monensin + infected by T. gondii (TG_Monensin), and hBMECs infected by T. gondii (TG_only), compared to control hBMECs. The red horizontal line represents a cut-off value of $p<0.05$ and the vertical dash red lines represent the $\log _{2}$ FC cut-off value of $\leq-1$ (downregulated) or $\geq 1$ (upregulated). Generally, more upregulated genes passed both cut-off values than down-regulated genes. (D-F) Correlation heatmaps of the top 30 most significant DEGs following treatment with monensin, following treatment + infection, and following infection, compared to control. The rows represent DEGs and the columns represent the 12 examined samples. The dendrogram shows a high intra-sample correlation between each group for all groups. Genes labelled as NA (not available) denote unpublished genes or uncharacterized non-coding RNA genes without annotation.

\subsubsection{Control vs. TG_only}

The overall expression profile of hBMECs infected with T. gondii (TG_only) for $24 \mathrm{~h}$ showed that more genes were upregulated than downregulated (Figure $6 \mathrm{C}$ ). The total number of genes that passed selection criteria was 902 genes, with 353 of them were upregulated while 549 were downregulated. Among the 902 DEGs, 454 were without annotation (NA), while the remaining 448 were annotated. For the annotated genes, 345 of them were upregulated, whereas 103 were downregulated. The top 30 
most significant DEGs from TG_only samples are listed in Table S8. Among these, the most highly significant upregulated gene with annotation was 'epithelial cell adhesion molecule' (EPCAM) with a $\log _{2}$ FC of 2.249. The top 30 upregulated and downregulated genes are listed in Tables S9 and S10, respectively. Among these, the most highly upregulated gene with annotation was 'metallothionein $1 \mathrm{M}^{\prime}(M T 1 M)$ with a $\log _{2} \mathrm{FC}$ of 4.414 and the most highly downregulated gene with annotation was 'microRNA 29a' (MIR29A) with a $\log _{2}$ FC of -3.201 . Figure $6 \mathrm{~F}$ depicts the heatmap showing a correlation between samples based on the top 30 most significant DEGs from $24 \mathrm{~h} \mathrm{~T}$. gondii infection.

\subsection{Gene Ontology (GO) and Pathway Analysis}

Here, the transcriptional changes in hBMECs in response to treatment with $0.1 \mu \mathrm{M}$ monensin (Monensin_only), T. gondii infection (TG_only), and T. gondii infection plus treatment with $0.1 \mu \mathrm{M}$ monensin (TG_Monensin) were investigated. For this analysis, only annotated genes were considered. Out of 112 downregulated genes, only $65(58.03 \%)$ were annotated, whereas 171 out of $368(46.47 \%)$ upregulated genes were annotated in the Monensin_only group. Regarding Control vs. TG_Monensin, 109 out of 134 (81.34\%) downregulated genes were annotated, and 201 out of 290 (69.31\%) up-regulated genes were annotated. For Control vs. TG_only, 103 out of 353 (29.18\%) downregulated genes were annotated, but 345 out of $549(62.84 \%)$ upregulated genes were annotated.

\subsubsection{Control vs. Monensin_only}

Of the 65 annotated downregulated genes, 54 were recognized by PANTHER database and the GO terms belonging to the categories of biological process (BP), molecular function (MF), cellular compartment (CC) are presented in Figure 7A-C, respectively. Overall, 54 genes matched 11 GO terms of BP, including cellular process, metabolic process, cellular component organization or biogenesis, biological regulation, response to stimulus, developmental process, multicellular organismal process, localization, reproduction, rhythmic process, and locomotion. Using PANTHER Overrepresentation Test with the default setting (Fisher's Exact with FDR multiple test correction) for PANTHER GO-Slim Biological Process, 11 out of 92 BPs were remarkably enriched (FDR < 0.05). These included DNA metabolic process, cell cycle, regulation of cell cycle, mitosis, nucleobase-containing compound metabolic process, DNA replication, chromosome segregation, organelle organization, cell proliferation, chromatin organization, and metabolic process. The downregulated genes also matched to four MF terms (binding, catalytic activity, receptor activity and structural molecule activity). The PANTHER Overrepresentation Test identified DNA helicase activity, helicase activity, kinase regulator activity, nucleic acid binding and binding as overrepresented MFs. In the CC ontology, downregulated genes matched six CC terms (cell part, organelle, macromolecular complex, membrane, extracellular matrix and extracellular region). The PANTHER Overrepresentation Test identified the nucleus as the only overrepresented CC.

Of the 171 annotated upregulated genes, 148 were recognized by the PANTHER database and the highly enriched terms for three GO categories (BP, MF, CC) are presented in Figure 7A-C, respectively. Overall, these genes matched to $12 \mathrm{BP} 12$ terms (cellular process, metabolic process, localization, cellular component organization or biogenesis, response to stimulus, biological regulation, developmental process, immune system process, multicellular organismal process, reproduction, rhythmic process, cell killing), six MFs terms (catalytic activity, binding, transporter activity, receptor activity, signal transducer activity, structural molecule activity) and six CC terms (cell part, organelle, membrane, macromolecular complex, extracellular region, extracellular matrix). The PANTHER Overrepresentation Test (FDR < 0.05) identified 10 significantly enriched BP terms (steroid metabolic process, protein targeting, protein transport, vesicle-mediated transport, intracellular protein transport, protein localization, transport, localization, single-multicellular organism process, multicellular organismal process), one MF term (SNAP receptor activity), and six CC terms (vesicle coat, SNARE complex, Golgi apparatus, cytoplasmic membrane-bounded vesicle, endoplasmic reticulum, cytoplasm). 


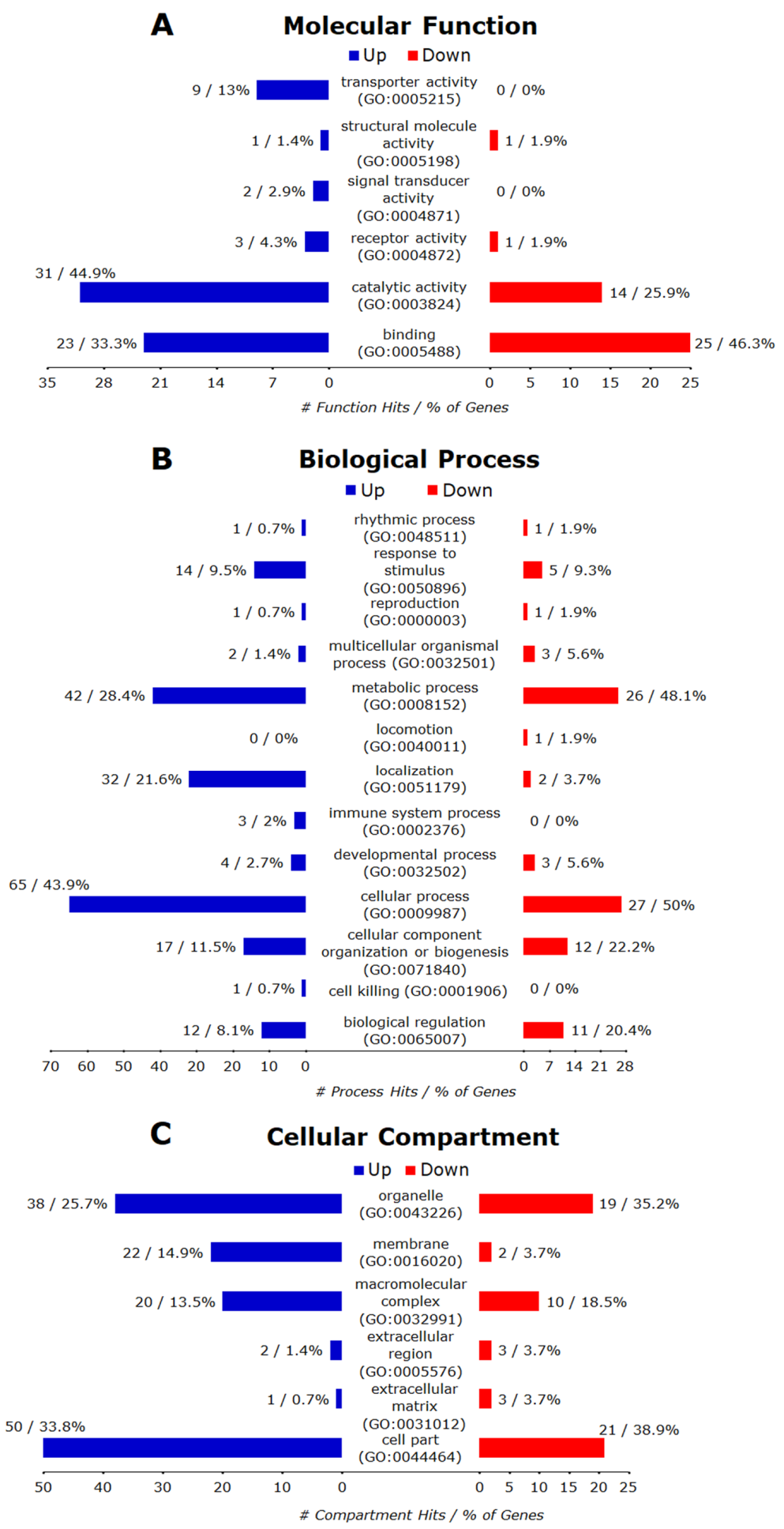

Figure 7. PANTHER GO-Slim gene ontology hits for significant DEGs due to treatment with $0.1 \mu \mathrm{M}$ monensin for $24 \mathrm{~h}$ (Monensin_only). (A) The down-regulated genes matched four MF terms and the up-regulated genes matched six MF terms. The PANTHER Overrepresentation Test (FDR < 0.05) identified five and one overrepresented MF terms for down- and up-regulated genes, respectively. (B) The down-regulated genes matched 11 BPs and the up-regulated genes matched 12 BPs. The PANTHER Overrepresentation Test (FDR < 0.05) identified 11 and 10 overrepresented BPs for down- and up-regulated genes, respectively. (C) The down-regulated genes matched six CCs while up-regulated genes matched six CCs. The PANTHER Overrepresentation Test (FDR < 0.05) identified one and six overrepresented CC terms for down- and up-regulated genes, respectively. 
All 236 annotated DEGs in the filtered list $\left(\log _{2} \mathrm{FC} \leq-1\right.$ or $\geq 1$; adjusted $p$-value $\left.<0.05\right)$ were submitted to the Reactome Pathways Analysis tool (Available online: https://reactome.org/ PathwayBrowser/\#TOOL=AT) together with their $\log _{2}$ FC values. Out of these, 132 were recognized by the Reactome database and considered for pathway analysis. All 23 pathways linked with the submitted genes with FDR $<0.05$ are listed in Table 1. The table includes Reactome pathway identifiers, pathway name, the number of matched genes, plus the pathway expression representation, which was calculated from the average $\log _{2}$ FC of the matched genes. Overall, 17 pathways were upregulated, whereas another six were downregulated. The most highly upregulated pathway was 'PERK regulates gene expression' (R-HSA-381042) with an average $\log _{2}$ FC of 2.165, but the most downregulated pathway was 'G0 and early G1' (R-HSA-1538133) with an average $\log _{2}$ FC of -1.179 . Table 2 lists pathways related to Wnt signalling, including their pathway's identifiers, the number of matched genes, FDR values and pathway expression representation by an average of $\log _{2}$ FC.

Table 1. All 23 Reactome pathways affected by $24 \mathrm{~h}$ of $0.1 \mu \mathrm{M}$ monensin treatment (Monensin_only) with FDR $<0.05$.

\begin{tabular}{|c|c|c|c|}
\hline Pathway ID & Pathway Name & Genes Found/Total & Average $\log _{2}$ FC \\
\hline R-HSA-381119 & Unfolded protein response (UPR) & $31 / 160$ & 1.440 \\
\hline R-HSA-381070 & IRE1alpha activates chaperones & $24 / 110$ & 1.273 \\
\hline R-HSA-381038 & $\mathrm{XBP} 1(\mathrm{~S})$ activates chaperone genes & $21 / 106$ & 1.293 \\
\hline R-HSA-1655829 & Regulation of cholesterol biosynthesis by SREBP (SREBF) & $19 / 86$ & 1.279 \\
\hline R-HSA-2426168 & Activation of gene expression by SREBF (SREBP) & $16 / 70$ & 1.295 \\
\hline R-HSA-199977 & ER to Golgi anterograde transport & $17 / 163$ & 1.318 \\
\hline R-HSA-8957322 & Metabolism of steroids & $24 / 321$ & 1.302 \\
\hline R-HSA-446203 & Asparagine N-linked glycosylation & $26 / 420$ & 1.301 \\
\hline R-HSA-381183 & ATF6 (ATF6-alpha) activates chaperone genes & $6 / 15$ & 1.766 \\
\hline R-HSA-381033 & ATF6 (ATF6-alpha) activates chaperones & $6 / 17$ & 1.766 \\
\hline R-HSA-6807878 & COPI-mediated anterograde transport & $12 / 106$ & 1.316 \\
\hline R-HSA-948021 & Transport to the Golgi and subsequent modification & $17 / 218$ & 1.318 \\
\hline R-HSA-191273 & Cholesterol biosynthesis & $10 / 72$ & 1.373 \\
\hline R-HSA-392499 & Metabolism of proteins & $75 / 2432$ & 0.969 \\
\hline R-HSA-204005 & COPII-mediated vesicle transport & $9 / 76$ & 1.381 \\
\hline R-HSA-6811442 & Intra-Golgi and retrograde Golgi-to-ER traffic & $14 / 217$ & 1.127 \\
\hline R-HSA-176974 & Unwinding of DNA & $4 / 12$ & -1.121 \\
\hline R-HSA-381042 & PERK regulates gene expression & $6 / 38$ & 2.165 \\
\hline R-HSA-1362300 & $\begin{array}{l}\text { Transcription of E2F targets under negative control by } \\
\text { p107 (RBL1) and p130 (RBL2) in complex with HDAC1 }\end{array}$ & $4 / 20$ & -1.096 \\
\hline R-HSA-1538133 & G0 and early G1 & $5 / 38$ & -1.179 \\
\hline R-HSA-1640170 & Cell cycle & $25 / 681$ & -0.763 \\
\hline R-HSA-6811438 & Intra-Golgi traffic & $5 / 48$ & 1.558 \\
\hline R-HSA-5693554 & $\begin{array}{l}\text { Resolution of D-loop structures through } \\
\text { synthesis-dependent strand annealing (SDSA) }\end{array}$ & $4 / 30$ & -1.129 \\
\hline
\end{tabular}

Note: Greyed rows indicate down-regulated pathways.

Table 2. Differentially expressed genes related to Wnt signaling (Monensin_only).

\begin{tabular}{ccccc}
\hline Pathway ID & Pathway Name & $\begin{array}{c}\text { Entities } \\
\text { Found/Total }\end{array}$ & $\begin{array}{c}\text { Average } \\
\mathbf{L o g}_{2} \text { FC }\end{array}$ & $\begin{array}{c}\text { FDR } \\
\text { Values }\end{array}$ \\
\hline $\begin{array}{l}\text { R-HSA-201681 } \\
\text { Matched Genes }\end{array}$ & TCF-dependent signalling in response to Wnt & $2 / 216$ & 1.232 & 0.918 \\
R-HSA-195721 & HIST1H2AJ; HIST1H3F & & \\
Matched Genes & Signalling by Wnt $2 / 330$ & 1.232 & 0.987 \\
\hline
\end{tabular}

\subsubsection{Control vs. TG_Monensin}

Of 109 annotated downregulated genes, 101 were recognized by the PANTHER database and the hits for all GO annotations (BP, MF, CC) and their major categories were presented in Figure 8A-C, respectively. Overall, these downregulated genes matched $10 \mathrm{BP}$ terms (biological adhesion, biological regulation, cellular component organization or biogenesis, cellular process, developmental process, localization, metabolic process, multicellular organismal process, reproduction, response to stimulus), 
three MF terms (binding, catalytic activity, structural molecule activity), and six CC terms (cell part, extracellular matrix, extracellular region, macromolecular complex, membrane, organelle). Further analysis with the PANTHER Overrepresentation Test (FDR $<0.05)$ revealed 25 significantly enriched BP terms (Table 3), nine significantly enriched MF terms (DNA binding, nucleic acid binding, DNA helicase activity, helicase activity, binding, single-stranded DNA binding, microtubule binding, microtubule motor activity, motor activity), and 12 significantly enriched CC terms (chromosome, nucleus, nuclear chromosome, organelle, protein-DNA complex, nucleoplasm, intracellular, cell part, microtubule, plasma membrane, membrane, cytoskeleton).

Of 201 annotated up-regulated genes, 173 were recognized by the PANTHER database and the hits for all GO annotations (BP, MF, CC) and their major categories are presented in Figure 8A-C, respectively. Overall, these up-regulated genes matched to $11 \mathrm{BP}$ terms (biological regulation, cellular component organization or biogenesis, cellular process, developmental process, immune system process, localization, locomotion, metabolic process, multicellular organismal process, reproduction, response to stimulus), seven MF categories (antioxidant activity, binding, catalytic activity, receptor activity, signal transducer activity, structural molecule activity, transporter activity), and six CC terms (cell part, extracellular matrix, extracellular region, macromolecular complex, membrane, organelle). Further analysis with the PANTHER Overrepresentation Test $(F D R<0.05)$ found nine significantly enriched BPs (protein glycosylation, vesicle-mediated transport, protein transport, protein localization, intracellular protein transport, transport, localization, single-multicellular organism process, multicellular organismal process) and nine significantly enriched CC terms (vesicle coat, SNARE complex, Golgi apparatus, cytoplasmic membrane-bounded vesicle, endoplasmic reticulum, nuclear outer membrane-endoplasmic reticulum membrane network, cytoplasm, organelle, intracellular), but no significantly enriched MF terms.

All 310 DEGs with a symbol from the filtered $\operatorname{list}\left(\log _{2} \mathrm{FC} \leq-1\right.$ or $\geq 1$; adjusted $p$-value $\left.<0.05\right)$ were submitted to the Reactome pathways analysis tool together with their $\log _{2} \mathrm{FC}$ values. Out of these, only 180 were recognized by the Reactome database and considered for pathway analysis. Pathways linked with the submitted genes with FDR $<0.05$ are listed in Table 4. Overall, the most up-regulated pathway was 'PERK regulates gene expression' (R-HSA-381042) with an average $\log _{2}$ FC of 2.065, whereas the most down-regulated pathway was 'Resolution of D-loop structures through Holliday junction intermediates' (R-HSA-5693568) and 'Resolution of D-Loop structures' (R-HSA-5693537), both with an average $\log _{2}$ FC of -1.239 . Table 5 lists pathways related to Wnt signalling plus their pathway's identifiers, the number of matched genes, FDR values, and pathway expression representation by an average of $\log _{2} \mathrm{FC}$.

Table 3. All 25 significantly enriched biological processes from 101 annotated upregulated genes, sorted with the most significant at the top (TG_only).

\begin{tabular}{ccc}
\hline PANTHER GO-Slim Biological Process (PO) & $\begin{array}{c}\text { No. of Matched } \\
\text { Genes }\end{array}$ & FDR \\
\hline DNA metabolic process (GO:0006259) & 23 & $5.44 \times 10^{-16}$ \\
Nucleobase-containing compound metabolic process (GO:0006139) & 38 & $1.04 \times 10^{-7}$ \\
Cell cycle (GO:0007049) & 19 & $1.22 \times 10^{-7}$ \\
Chromosome segregation (GO:0007059) & 9 & $1.51 \times 10^{-7}$ \\
Mitosis (GO:0007067) & 11 & $1.1 \times 10^{-6}$ \\
DNA repair (GO:0006281) & 9 & $6.88 \times 10^{-6}$ \\
Organelle organisation (GO:0006996) & 21 & $1.04 \times 10^{-5}$ \\
Regulation of cell cycle (GO:0051726) & 9 & $1.23 \times 10^{-5}$ \\
DNA replication (GO:0006260) & 8 & $3.44 \times 10^{-5}$ \\
Nitrogen compound metabolic process (GO:0006807) & 30 & $4.27 \times 10^{-5}$ \\
Chromatin organisation (GO:0006325) & 9 & $1.87 \times 10^{-4}$ \\
Metabolic process (GO:0008152) & 48 & $6.35 \times 10^{-4}$ \\
\hline
\end{tabular}


Table 3. Cont.

\begin{tabular}{ccc}
\hline PANTHER GO-Slim Biological Process (PO) & $\begin{array}{c}\text { No. of Matched } \\
\text { Genes }\end{array}$ & FDR \\
\hline Primary metabolic process (GO:0044238) & 41 & $1.18 \times 10^{-3}$ \\
DNA recombination (GO:0006310) & 4 & $2.59 \times 10^{-3}$ \\
Cellular component organisation (GO:0016043) & 22 & $3.08 \times 10^{-3}$ \\
Cell communication (GO:0007154) & 2 & $3.69 \times 10^{-3}$ \\
Cellular component organisation or biogenesis (GO:0071840) & 22 & $5.55 \times 10^{-3}$ \\
Regulation of gene expression, epigenetic (GO:0040029) & 4 & $6.23 \times 10^{-3}$ \\
Chromatin assembly (GO:0031497) & 3 & 0.0146 \\
Signal transduction (GO:0007165) & 2 & 0.0147 \\
Meiosis (GO:0007126) & 3 & 0.0433 \\
Multicellular organismal process (GO:0032501) & 1 & 0.0459 \\
Single-multicellular organism process (GO:0044707) & 1 & 0.0469 \\
Cell surface receptor signalling pathway (GO:0007166) & 0 & 0.0485 \\
Cell proliferation (GO:0008283) & 3 & 0.0487 \\
\hline
\end{tabular}

Table 4. The top 30 out of 55 Reactome pathways affected by T. gondii infection and treatment with $0.1 \mu \mathrm{M}$ monensin (TG_Monensin). Among the 310 genes submitted, only 180 were recognized by the Reactome database and considered for analysis.

\begin{tabular}{|c|c|c|c|}
\hline Pathway ID & Pathway Name & $\begin{array}{l}\text { No. of Matched } \\
\text { Genes }\end{array}$ & $\begin{array}{l}\text { Average } \\
\log _{2} \text { FC }\end{array}$ \\
\hline R-HSA-381038 & $\mathrm{XBP1}(\mathrm{S})$ activates chaperone genes & $27 / 106$ & 1.399 \\
\hline R-HSA-381070 & IRE1alpha activates chaperones & $30 / 110$ & 1.373 \\
\hline R-HSA-381119 & Unfolded protein response (UPR) & $38 / 160$ & 1.469 \\
\hline R-HSA-69278 & Cell cycle, mitotic & $43 / 569$ & -1.000 \\
\hline R-HSA-1640170 & Cell cycle & $47 / 681$ & -1.017 \\
\hline R-HSA-1538133 & G0 and early G1 & $10 / 38$ & -1.091 \\
\hline R-HSA-446203 & Asparagine N-linked glycosylation & $31 / 420$ & 1.307 \\
\hline R-HSA-199977 & ER to Golgi anterograde transport & $18 / 163$ & 1.343 \\
\hline R-HSA-453279 & Mitotic G1-G1/S phases & $17 / 173$ & -1.091 \\
\hline R-HSA-69620 & Cell cycle Checkpoints & $22 / 279$ & -1.017 \\
\hline R-HSA-381183 & ATF6 (ATF6-alpha) activates chaperone genes & $6 / 15$ & 1.612 \\
\hline R-HSA-6811442 & Intra-Golgi and retrograde Golgi-to-ER traffic & $19 / 217$ & 0.809 \\
\hline R-HSA-6807878 & COPI-mediated anterograde transport & $13 / 106$ & 1.362 \\
\hline R-HSA-381033 & ATF6 (ATF6-alpha) activates chaperones & $6 / 17$ & 1.612 \\
\hline R-HSA-392499 & Metabolism of proteins & $92 / 2432$ & 0.796 \\
\hline R-HSA-948021 & Transport to the Golgi and subsequent modification & $18 / 218$ & 1.343 \\
\hline R-HSA-1362300 & $\begin{array}{l}\text { Transcription of E2F targets under negative control by p107 } \\
\text { (RBL1) and p130 (RBL2) in complex with HDAC1 }\end{array}$ & $6 / 20$ & -1.054 \\
\hline R-HSA-6811434 & COPI-dependent Golgi-to-ER retrograde traffic & $12 / 106$ & 0.447 \\
\hline R-HSA-141424 & Amplification of signal from the kinetochores & $11 / 94$ & -0.858 \\
\hline R-HSA-141444 & $\begin{array}{l}\text { Amplification of signal from unattached kinetochores via a MAD2 } \\
\text { inhibitory signal }\end{array}$ & $11 / 94$ & -0.858 \\
\hline R-HSA-69618 & Mitotic spindle checkpoint & $11 / 110$ & -0.858 \\
\hline R-HSA-68886 & M Phase & $23 / 389$ & -0.912 \\
\hline R-HSA-2500257 & Resolution of sister chromatid cohesion & $12 / 133$ & -0.872 \\
\hline R-HSA-5693568 & $\begin{array}{c}\text { Resolution of D-loop structures through Holliday junction } \\
\text { intermediates }\end{array}$ & $6 / 36$ & -1.239 \\
\hline R-HSA-8856688 & Golgi-to-ER retrograde transport & $12 / 147$ & 0.447 \\
\hline R-HSA-5693537 & Resolution of D-Loop structures & $6 / 37$ & -1.239 \\
\hline R-HSA-381042 & PERK regulates gene expression & $6 / 38$ & 2.065 \\
\hline R-HSA-69206 & G1/S Transition & $12 / 150$ & -1.103 \\
\hline R-HSA-1362277 & $\begin{array}{l}\text { Transcription of E2F targets under negative control by DREAM } \\
\text { complex }\end{array}$ & $5 / 25$ & -1.070 \\
\hline R-HSA-774815 & Nucleosome assembly & $7 / 54$ & -1.108 \\
\hline
\end{tabular}


A

Molecular Function

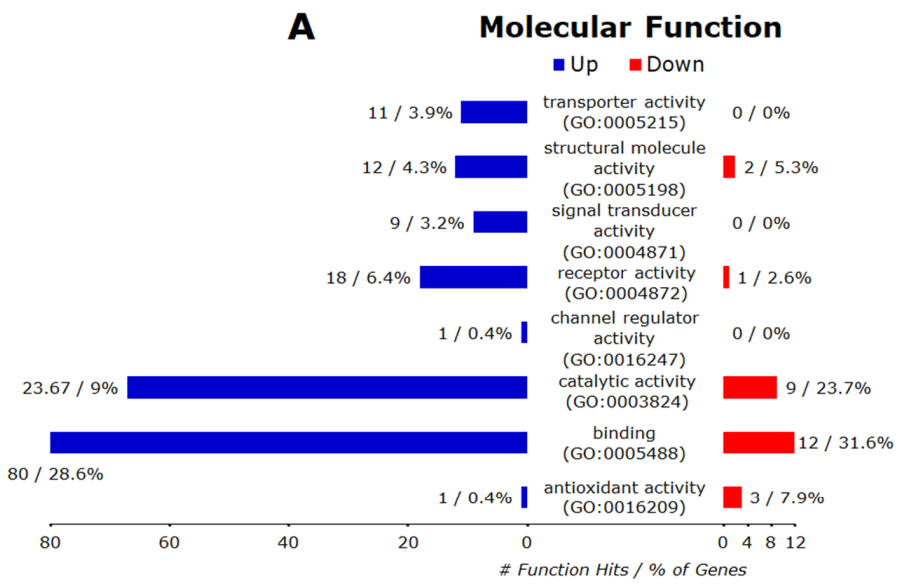

B

Biological Process
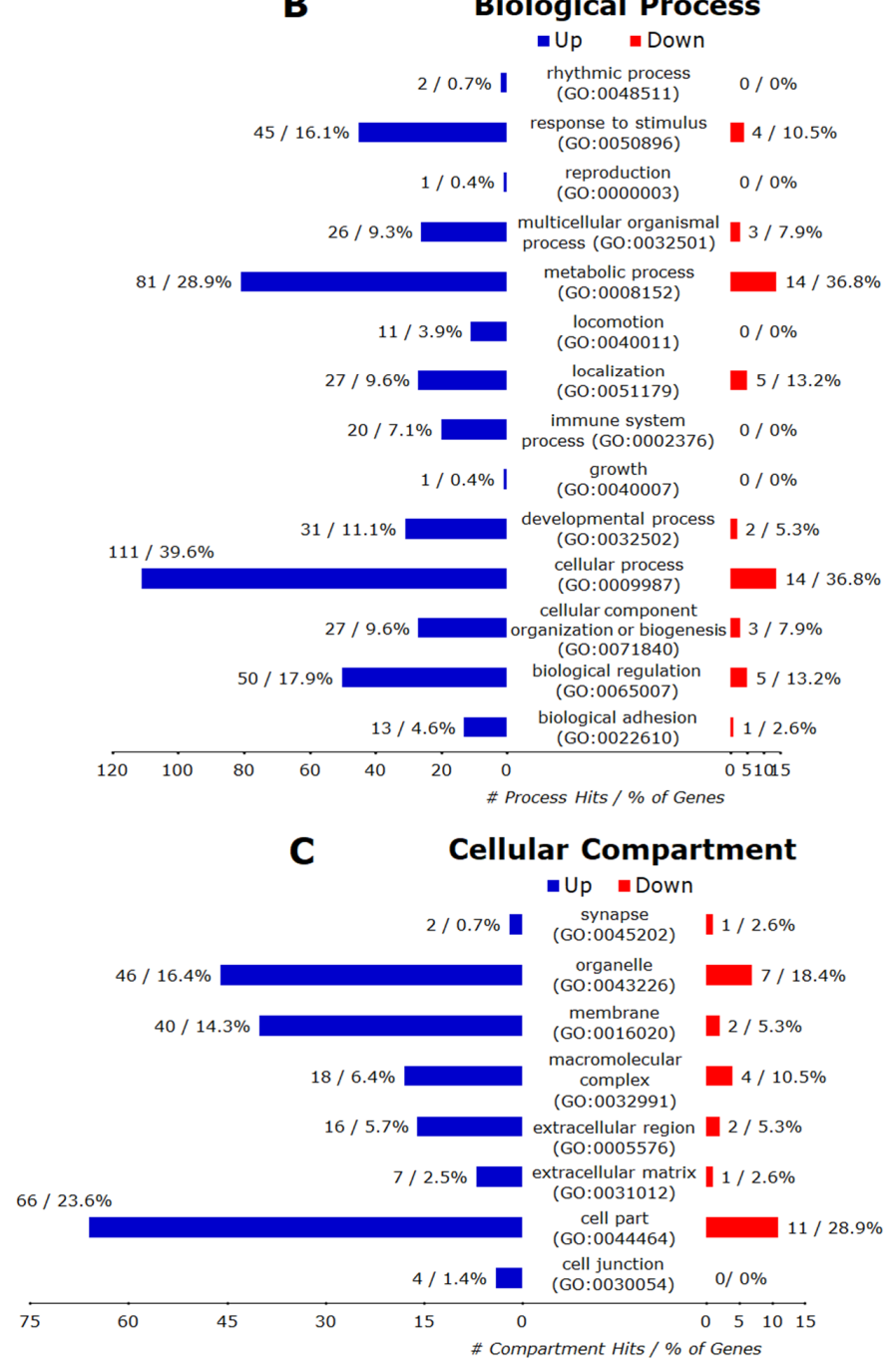

Figure 8. PANTHER GO-Slim gene ontology hits for significant DEGs due to T. gondii infection and treatment with monensin (TG_Monensin). (A) The down-regulated genes matched 3 MFs while the up-regulated genes matched seven MFs. The PANTHER Overrepresentation Test (FDR < 0.05) identified nine MFs down-regulated genes but none for up-regulated genes. (B) The down-regulated genes matched $10 \mathrm{BP}$ terms while the up-regulated genes matched 11 terms. The PANTHER Overrepresentation Test (FDR < 0.05) identified 25 and nine overrepresented BPs for down- and up-regulated genes, respectively. (C) The down-regulated genes matched six CC terms while the up-regulated genes matched six CC terms. The PANTHER Overrepresentation Test (FDR < 0.05) identified 12 and nine overrepresented CCs for down-regulated and up-regulated genes, respectively. 
Table 5. Expressed genes related to Wnt signaling (TG_Monensin) according to the Reactome classification.

\begin{tabular}{ccccc}
\hline \multirow{2}{*}{ Pathway ID } & Pathway Name & $\begin{array}{c}\text { Genes } \\
\text { Found/Total }\end{array}$ & $\begin{array}{c}\text { Average Log } \\
\text { FC }\end{array}$ & $\begin{array}{c}\text { FDR } \\
\text { Values }\end{array}$ \\
\hline R-HSA-195721 & Signalling by Wnt & $5 / 330$ & -1.207 & 0.911 \\
Matched Genes & HIST1H2BM; HIST1H2AJ; HIST1H3F; HIST1H3G; HIST1H2BE; HIST1H3B; HIST1H2AB \\
R-HSA-201681 & TCF-dependent signalling in response to Wnt & $5 / 216$ & -1.207 & 0.617 \\
Matched Genes & HIST1H2BM; HIST1H2AJ; HIST1H3F; HIST1H3G; HIST1H2BE; HIST1H3B; HIST1H2AB \\
\hline & Note: HIST1H2BM/HIST1H2BE and HIST1H3B/HIST1H3F are duplicated genes.
\end{tabular}

\subsubsection{Control vs. TG_only}

Of 103 annotated down-regulated genes, only 38 were recognized by the PANTHER database, and the hits for all GO annotations (BP MF, CC) and their major categories were presented in Figure 9A-C. Overall, these upregulated genes matched to $9 \mathrm{BP}$ terms (cellular process, metabolic process, localization, biological regulation, response to stimulus, cellular component organization or biogenesis, multicellular organismal process, developmental process, biological adhesion), five MF terms (binding, catalytic activity, transporter activity, signal transducer activity, receptor activity) and seven CC terms (cell part, organelle, macromolecular complex, membrane, extracellular region, synapse, extracellular matrix). Further analysis with the PANTHER Overrepresentation Test found no significantly enriched BP, MF or CC (FDR $<0.05)$.

Of 345 annotated up-regulated genes, only 280 were recognized by the PANTHER database and the hits for all GO annotations (BP MF, CC) and their major categories were presented in Figure 9A-C, respectively. Overall, these up-regulated genes matched to $14 \mathrm{BP}$ terms (biological adhesion, biological regulation, cellular component organization or biogenesis, cellular process, developmental process, growth, immune system process, localization, locomotion, metabolic process, multicellular organismal process, reproduction, response to stimulus, rhythmic process), eight MF terms (antioxidant activity, binding, catalytic activity, channel regulator activity, receptor activity, signal transducer activity, structural molecule activity, transporter activity) and eight CC terms (cell junction, cell part, extracellular matrix, extracellular region, macromolecular complex, membrane, organelle, synapse). Further analysis with the PANTHER Overrepresentation Test found BP to be significantly enriched, but there were no significantly enriched MF or CC (FDR $<0.05)$.

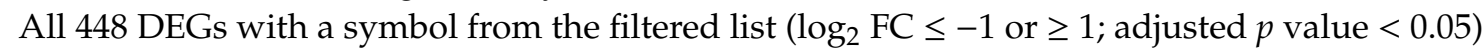
were submitted to Reactome's 'Pathways Analysis' tool together with their $\log _{2}$ FC values. Out of these, only 232 were recognized by the Reactome database and considered for pathway analysis. Pathways linked to the submitted genes with FDR $<0.05$ are listed in Table 6. Surprisingly, even though there were more genes submitted compared to Monensin_only, only four pathways passed the corrected overrepresentation probability test (FDR < 0.05). All four pathways were upregulated, with the most highly upregulated pathway being 'Interferon alpha/beta signalling' (R-HSA-909733) with an average $\log _{2}$ FC of 1.57. Table 7 lists pathways related to the Wnt signalling plus their pathway's identifiers, the number of matched genes, FDR values, and pathway expression representation by an average of $\log _{2}$ FC.

Table 6. Reactome pathways affected by $T$. gondii infection (TG_only) with FDR $<0.05$.

\begin{tabular}{cccc}
\hline Pathway ID & Pathway Name & Genes Found/Total & Average Log $_{2}$ FC \\
\hline R-HSA-909733 & Interferon alpha/beta signalling & $38 / 184$ & 1.611 \\
R-HSA-913531 & Interferon Signalling & $50 / 388$ & 1.532 \\
R-HSA-1280215 & Cytokine Signalling in the Immune system & $72 / 1051$ & 1.535 \\
R-HSA-877300 & Interferon gamma signalling & $23 / 250$ & 1.359 \\
\hline
\end{tabular}


A

Molecular Function

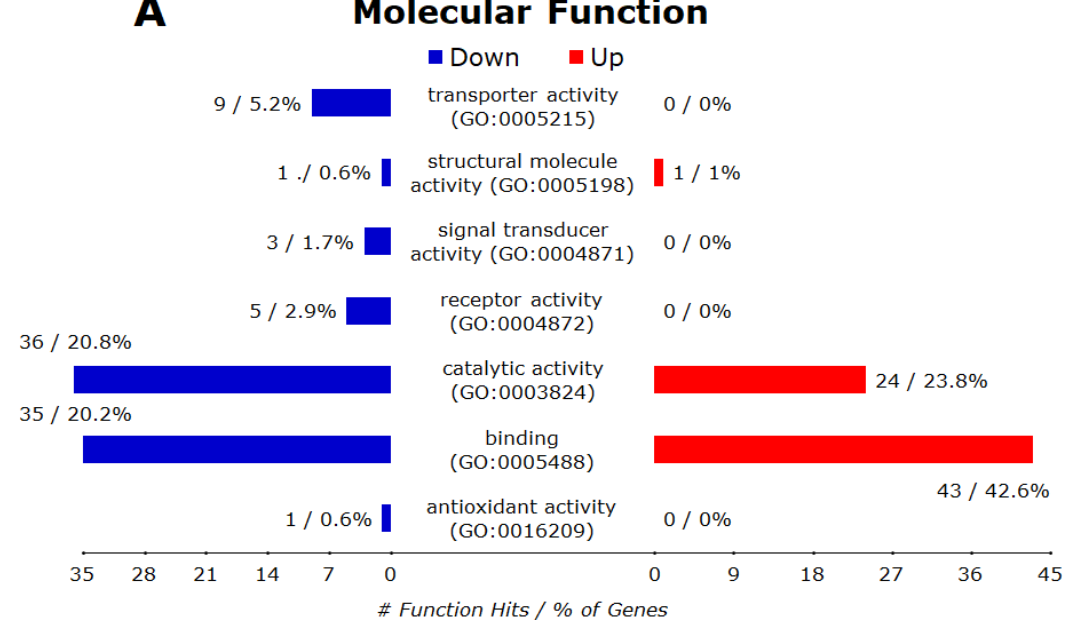

B

Biological Process

- Down u Up

$18 / 10.4 \% \square \begin{gathered}\text { response to stimulus } \\ \text { (GO:0050896) }\end{gathered}$

$9 / 8.9 \%$

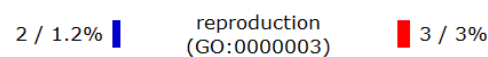

$3 / 1.7 \%$ multicellular organismal $\mid 1 / 1 \%$

$51 / 29.5 \%$

metabolic process

(GO:0008152)

$0 / 0 \%$

$1 / 0.6 \%$

locomotion

(GO:0040011)

$30 / 17.3 \%$

localization

(GO:0051179)

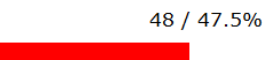

$0 / 0 \%$

$5 / 2.9 \%$

immune system proces
(GO:0002376)

$5 / 5 \%$

$6 / 3.5 \%$

developmental proces

(GO:0032502)
| $2 / 2 \%$

$76 / 43.9 \%$

cellular process (GO:0009987)

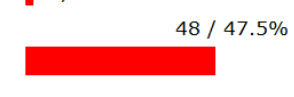

cellular component rganization or biogenesis (GO:0071840)

$18 / 10.4 \%$

biological regulation (GO:0065007)

$19 / 11 \%$ biological adhesion

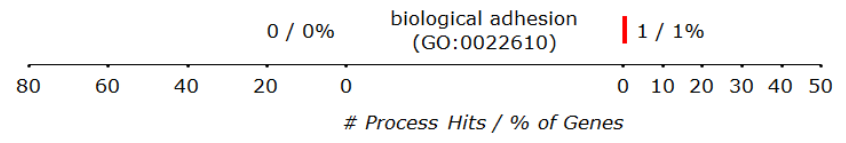

C

Cellular Compartment

- Down Uup

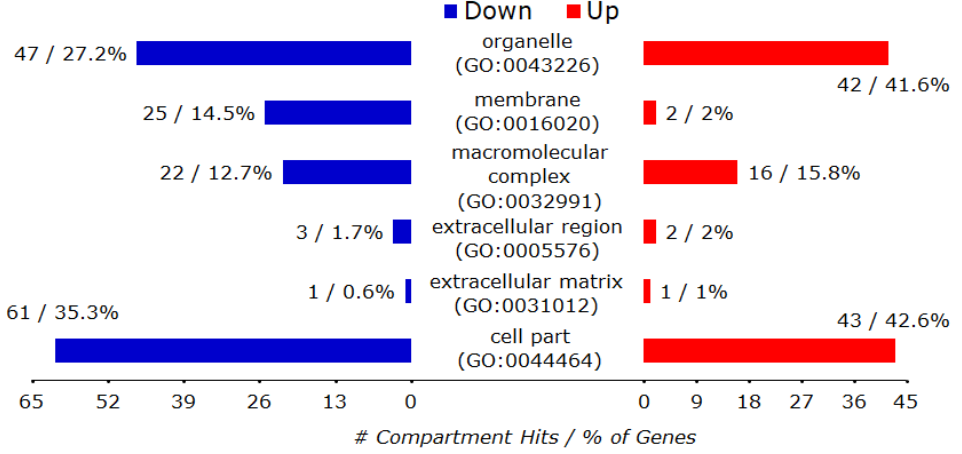

Figure 9. PANTHER GO-Slim gene ontology hits for significant DEGs due to T. gondii infection (TG_only). (A) The down-regulated genes matched five MF terms while the up-regulated genes matched eight MFs. No overrepresented MF was identified from the PANTHER Overrepresentation 
Test (FDR < 0.05) for both sets of genes. (B) The down-regulated genes matched nine PBs while the up-regulated genes matched 14 BPs. The PANTHER Overrepresentation Test (FDR < 0.05) identified two overrepresented BPs from up- but none for down-regulated genes. (C) The down-regulated genes matched seven CC terms while the up-regulated genes matched eight CC terms. No overrepresented $\mathrm{CC}$ was identified from the PANTHER Overrepresentation Test $(\mathrm{FDR}<0.05)$ for both sets of genes.

Table 7. Differentially expressed genes involved in Wnt signaling (TG_only) according to the Reactome classification.

\begin{tabular}{ccccc}
\hline Pathway ID & Pathway Name & $\begin{array}{c}\text { Genes } \\
\text { Found/Total }\end{array}$ & $\begin{array}{c}\text { Average } \text { Log }_{2} \\
\text { FC }\end{array}$ & $\begin{array}{c}\text { FDR } \\
\text { Values }\end{array}$ \\
\hline R-HSA-195721 & Signalling by Wnt & $7 / 330$ & 1.303 & 0.937 \\
Matched Genes & HIST1H4B; FZD7; HIST1H2BG; HIST2H3D; PLCB1; GNG11; HIST1H2AB & \\
R-HSA-201681 & TCF-dependent signalling in response to Wnt & 4/216 & 1.279 & 0.939 \\
Matched Genes & \multicolumn{2}{c}{ HIST1H4B; HIST1H2BG; HIST2H3D; HIST1H2AB } \\
R-HSA-3858494 & Beta-catenin independent Wnt signalling & 3/165 & 1.335 & 0.923 \\
Matched Genes & \multicolumn{2}{c}{ FZD7; PLCB1; GNG11 } \\
\hline
\end{tabular}

\section{Discussion}

In this study, we utilized microarray analysis to evaluate the transcriptome profile of T. gondii-infected hBMECs treated with the Wnt inhibitor monensin. First, we investigated the effect of Wnt inhibition by monensin on the in vitro growth of T. gondii inside hBMECs and microglia cells. T. gondii growth inhibition was evidenced by the significant reduction in the number of tachyzoites (Figure 1) and the relative similarity in cell density levels (Figure 2) between infected and non-infected cultures. Our data showed that parasite inhibition increased as the concentration of monensin increased (i.e. dose-dependent) and that monensin at $0.1 \mu \mathrm{M}$ concentration significantly decreased the growth of $T$. gondii tachyzoites. Consistent with this, it has been reported that monensin reduced T. gondii growth in human foreskin fibroblasts [26,27]. Our result also fits within the effective range of monensin concentrations $(0.01$ and $0.1 \mu \mathrm{M})$ reported against human cytomegalovirus (HCMV) [14]. Interestingly, monensin was more effective in inhibiting T. gondii growth in hBMECs compared to microglia cells. Moreover, in the presence of monensin treatment, infected and noninfected hBMECs exhibited significant reduction in their growth rates. However, the differential variation in the magnitude of cell growth reduction between the two cell lines suggests that monensin had more cytotoxic effect on hBMECs compared to microglia cells. These findings were the basis for choosing hBMECs to determine the effects of monensin on host gene transcription in the context of T. gondii infection.

Next, we determined the effect of $T$. gondii with or without monensin treatment on the integrity of cerebrovascular endothelial cell monolayer. Our findings showed that T. gondii infection can compromise the integrity of BBB endothelial cells compared to uninfected cells $(p<0.05$; Figure 3). Consistent with our finding, T. gondii was reported to compromise the cell integrity to facilitate a paracellular migration route across the BBB [28]. Cellular barrier function is mediated through intercellular tight junction (TJ) proteins, which can be dysregulated by T. gondii infection to increase barrier permeability and parasite crossing [29]. Alteration in the TJ function may occur either through a direct effect of $T$. gondii infection on the host cell cytoskeleton [30] or via the indirect action of T. gondii-induced proinflammatory cytokines, which can promote the opening of TJ that would in turn allow leukocytes to migrate across the endothelial barrier into cerebral tissue [31,32]. T. gondii-infected and treated hBMECs seemed to maintain their resistance, presumably due to the inhibitory effect of monensin on T. gondii growth, thereby protecting cells from cytoskeletal rearrangement associated with parasite invasion and growth, which compromises TJ and BBB integrity. Given the ability of this parasite to invade and use leukocytes as a Trojan-horse to migrate across the BBB [33], further studies should investigate how monensin may influence the effect of T. gondii on leukocytes' ability to cross 
the BBB. If monensin is effective in inhibiting T. gondii growth inside leukocytes, then this may thwart the parasite's ability to disseminate to the CNS tissue.

The exact mechanism of anti-T. gondii activity of monensin is unknown, however this compound seems to exert its action by directly interfering with one or more functions in the parasite. For example, osmotic swelling of the parasite [34], presumably due to its inability to regulate ionic homeostasis as a result of $\mathrm{Na}^{+}$influx [35], interference with vesicular trafficking [36], altering the parasite's cell cycle [26], or inducing nutrient stress and TgMSH-1-mediated autophagy [27], have all been suggested to contribute to T. gondii death. Interestingly, pre-treatment of hBMECs with monensin inhibited the susceptibility of hBMEC to gram-negative bacteria Citrobacter freundii [37] and Brucella abortus [38] invasion or survival in a dose-dependent manner. Monensin exhibits potent anticancer activity on several types of cancer, by inhibiting cell proliferation, cell cycle progression, and cell migration and by inducing apoptosis [39]. Monensin may also accomplish its anticancer effect by targeting multiple signaling pathways, particularly the epidermal growth factor receptor (EGFR) and Wnt signaling pathways $[39,40]$. Given these pervasive effects of monensin, it is possible that monensin exerts its anti-T. gondii effect, by altering the host response to infection in addition to its direct antiparasitic effect.

Therefore, we investigated whether the secondary effects of monensin on the host cells may contribute to parasite clearance. We performed microarray analysis to test the hypothesis that treatment with $0.1 \mu \mathrm{M}$ monensin modulates signalling pathways, such as the Wnt pathway, which in turn inhibits T. gondii infection and enhances hBMECs survival. The microarray quality control checks indicated that the datasets had acceptable quality. Variations within samples were minimal (Figure 4) and variations between samples were made comparable by RMA normalization (Figure S1). Our analysis revealed 480 DEGs in hBMECs treated with monensin compared to control untreated hBMECs. Since monensin induced significant changes in the gene transcription of treated cells, we wanted to investigate whether monensin also can cause the same changes in the presence of T. gondii infection. Interestingly, 424 DEGs were detected in T. gondii-infected hBMECs treated with monensin compared to control hBMECs. When we compared DEGs in monensin-treated uninfected cells to DEGs in monensin-treated T. gondii-infected cells, different levels of expression of specific genes were detected between the two groups, showing the differential response of treated cells in the presence or absence of infection. This finding is anticipated because a subset of these DEGs can be expected to be mechanistically involved in the infection process and thus their expression is unlikely to change in response to treatment per se. Interestingly, the TG_only group expression profile was distinct from the expression profiles of the other three groups (TG_Monensin group, Monensin_only group and Control_only group) as shown in the dendrogram (Figure 4A). This clustering is supported by the detection of 902 DEGs in hBMECs infected by T. gondii without monensin treatment (TG_only group), compared to 424 DEGs detected in infected and treated cells (TG_Monensin group). Such a significantly large number of DEGs in the TG_only group indicates that monensin must have attenuated the adverse impact of T. gondii infection on the treated cells, probably by interfering with signalling pathways essential for the parasite growth or via direct inhibition of the parasite proliferation, providing further support to the antiparasitic effect of monensin observed in Figures 1 and 2.

PANTHER GO enrichment analysis, used to functionally annotate all DEGs in each group, revealed similar terms in the three GO categories between the examined groups. Reactome pathway enrichment analysis of the DEGs of hBMECs treated with monensin revealed that monensin treatment downregulated pathways related to steroids, cholesterol and protein biosynthesis and their transport between endoplasmic reticulum (ER) and Golgi apparatus. Transcriptional analysis of T. gondii-infected hBMECs treated with monensin revealed a similar transcriptional pattern to the Monensin_only group. These results suggest that parasite inhibition may have been achieved through dysregulation of the biosynthesis of key nutrients in the host cells and/or inhibition of nutrient transport between cell organelles, limiting their transport into parasitophorous vacuole. This finding is consistent with a previous transcriptomic study of monensin-treated porcine kidney (PK)-15 cells, which revealed an overrepresentation of the downregulated genes involved in the biosynthesis of spliceosome, ribosome, 
and protein processing in ER, suggesting that monensin, via down-regulation of protein biosynthesis, can limit the parasite growth and proliferation [41].

Our data also showed that monensin administration modulated pathways related to DNA replication in the nucleus and cell cycle. In our study, we have identified 39 significantly upregulated pathways related to DNA replication, cell cycle and cell division in the TG_Monensin group compared to only three significantly upregulated pathways from the Monensin_only transcriptome data. The significantly increased number of these upregulated pathways in the TG_Monensin group is probably attributed to the dual effects of monensin and parasite infection on the host cell transcriptome. The effects of monensin on DNA synthesis, and repair of the parasite and the host cells, can be also inferred from the downregulated pathways identified in both the Monensin_only and the TG_Monensin groups. Some of the downregulated pathways were related to DNA repair mechanisms, such as resolution of D-loop structures through Holliday junction intermediates (R-HSA-5693568), resolution of D-loop structures (R-HSA-5693537), resolution of D-loop structures through synthesis-dependent strand annealing (R-HSA-5693554), and unwinding of DNA (R-HSA-176974). In addition, key DNA repair mechanisms related to molecular functions, such as DNA helicase activity (GO:0003678), helicase activity (GO:0004386), kinase regulator activity (GO:0019207), nucleic acid binding (GO:0003676) and binding (GO:0005488), were also identified as overrepresented (PANTHER Overrepresentation Test) from downregulated genes of the Monensin_only group.

Interestingly, both the Monensin_only and the TG_Monensin groups downregulated cell cycle-related pathways (e.g. cell cycle (R-HSA-1640170); G0 and early G1 (R-HSA-1538133) and transcription of E2F targets under negative control by p107/RBL1 and p130/RBL2 in complex with HDAC1 [R-HSA-1362300]) and upregulated PERK regulates gene expression (R-HSA-381042), which leads to cell cycle arrest in host cells. Monensin has been shown to arrest the parasite cell cycle process [26]. Thus, we can infer that monensin can inhibit both parasite and host cell cycle processes that ultimately lead to growth inhibition of T. gondii. This finding is consistent with the upregulation of the DNA damage-inducible transcript 3 (DDIT3), a key gene in the upregulated pathway, which encodes a stress-inducible transcription factor that induces cell cycle arrest and apoptosis in mammalian cells. Our finding is consistent with a previous study that showed that monensin induced DDIT3, a key inhibitor of the Wnt signalling pathway [42], in prostate cancer cells [43]. The key genes identified in those downregulated pathways are CCNA2, that controls both the G1/S and the G2/M transition phases of the cell cycle, and MYBL2, that functions as a physiological regulator of cell cycle progression. While there are no published data that show the inhibitory effect of monensin on $C C N A 2$, there are reports on monensin as a potent inhibitor of the MYB gene family that includes MYBL1 and MYBL2 [44-46].

The link between $T$. gondii and the Wnt signalling pathway has been already established. In one study, the Wnt pathway was found to be upregulated during T. gondii infection in brain cells [47]. In another study, the virulence factor ROP18 of T. gondii was found to inhibit the Wnt signalling pathway in neural stem cells [48]. In the present study, Wnt signalling and Wnt-related pathways were not significantly affected by treatment with $0.1 \mu \mathrm{M}$ monensin, rejecting the hypothesis that inhibition of $T$. gondii growth is largely mediated by the aberrant Wnt signalling in the host cells due to monensin treatment. All Wnt-related genes, such as Frizzled receptors, beta-catenin and protein kinase C (PKC) had modest expression after treatment. The same also applies to the transcriptome of TG_only and TG_Monensin groups. This finding suggests that monensin treatment did not affect the Wnt signalling pathway in hBMECs and that inhibition of T. gondii infection in hBMECs following monensin treatment was not achieved through direct blocking of the Wnt signalling pathway. Our study did however identify changes in the expression of genes related to other signal pathways involved in cell cycle control, apoptosis, and DNA synthesis and repair. These data further support the pleiotropic effects of monensin on multiple biological processes.

We were also interested in comparing host gene expression in uninfected to T. gondii-infected hBMECs in the absence of monensin treatment to better understand the types of host responses induced by infection. The transcriptome analysis of hBMECs infected with $T$. gondii for $24 \mathrm{~h}$ (TG_only) revealed 
significantly increased interferon-alpha/beta and gamma signalling-related pathways. This finding is consistent with previous transcriptome studies in murine macrophages [49] and human fibroblast cells [50], reaffirming the deregulation of the immune response following $T$. gondii infection of hBMECs. The increased expression of the MT1M gene was also reported previously [51], where MT1M upregulation has been suggested to contribute to the cellular homeostasis of transition metals, particularly zinc, during T. gondii infection. The down-regulation of microRNA 29a has also been reported in a mouse brain infected by the cystogenic strain (type II) [52], which is less virulent than the RH (type I) strain used in our study, suggesting that inhibition of microRNA 29a is common in T. gondii infection, independent of the parasite virulence.

\section{Conclusions}

We showed that the ionophore monensin, an inhibitor of the Wnt pathway, had a significant anti-T. gondii activity against the tachyzoite stage of $T$. gondii grown in hBMECs and microglia in vitro at $0.1 \mu \mathrm{M}$ concentration. We also showed an association between $T$. gondii infection and compromisation of the hBMEC integrity, which was restored by treatment with $0.1 \mu \mathrm{M}$ monensin. Transcriptome analysis provided evidence that treatment of hBMECs with monensin is accompanied by reduced expression of genes in pathways involved in steroids, cholesterol and protein biosynthesis and their transport between ER and Golgi apparatus. These data indicate that inhibition of T. gondii infection in hBMECs was not directly achieved through blocking Wnt signalling and that anti-T. gondii activity of monensin appears to be mediated independently of the Wnt pathway. Rather, our findings support the notion that parasite inhibition was in part due to inhibition of transport between organelles within cells and limiting nutrient transport into parasitophorous vacuole. In addition, monensin seems to inhibit the parasite growth through interference with cell cycle and DNA synthesis and repair mechanisms. More studies to elucidate how host-targeted effects of monensin on hBMECs inhibits T. gondii infection are warranted. Future work should also include experiments on the protein level to assess whether differential gene regulation is paralleled by similar proteomic changes.

Supplementary Materials: The following are available online at http://www.mdpi.com/2076-2607/8/6/842/s1, Figure S1. Raw and normalized expression measurements of the microarray data. Box and whiskers plots showing expression values of all samples pre- (A) and post-normalization (B). The plots consist of boxes with a central horizontal line that represents the median of the data and two tails (dotted vertical lines) that represent the upper and lower quartile. The variable lengths of the solid vertical lines show the heterogeneity in the distribution of the microarray files before implementing normalization. The samples were normalized using the widely used robust multiarray average (RMA) method, which considers background correction and variation between chipsets [21,22]. Normalization improved the overall distribution between samples and rendered the data more balanced for the accurate sample to sample comparison ahead of the analysis, Table S1. List of 19 DEGs induced in three groups (Monensin_only, TG_Monensin, and TG_only) compared to the control group, Table S2. The top 30 most significant DEGs detected in hBMECs after $24 \mathrm{~h}$ of $0.1 \mu \mathrm{M}$ monensin treatment (Monensin_only), Table S3. The top 30 upregulated genes detected in hBMECs treated with $0.1 \mu \mathrm{M}$ monensin (Monensin_only) sorted in a descending order, with the highest $\log _{2}$ FC at the top, Table S4. The top 30 downregulated genes detected in hBMECs treated with $0.1 \mu \mathrm{M}$ monensin (Monensin_only) sorted with the lowest $\log _{2}$ FC at the top, Table S5. The top 30 most significant DEGs detected in hBMECs infected with T. gondii and treated with $0.1 \mu \mathrm{M}$ monensin (TG_Monensin), Table S6. The top 30 upregulated genes detected in $0.1 \mu \mathrm{M}$ monensin treated and T. gondii-infected hBMECs (TG_Monensin) sorted with the highest $\log _{2}$ FC values at the top, Table S7. The top 30 downregulated genes detected in $0.1 \mu \mathrm{M}$ monensin treated and T. gondii-infected hBMECs (TG_Monensin) sorted with the lowest $\log _{2}$ FC values at the top, Table S8. The top 30 most significant DEGs induced in hBMECs $24 \mathrm{~h}$ after T. gondii infection (TG_only), Table S9. The top 30 upregulated genes detected in T. gondii-infected hBMECs (TG_only) sorted with the highest $\log _{2}$ FC at the top, Table S10. The top 30 downregulated genes detected in T. gondii-infected hBMECs (TG_only) sorted with the $\operatorname{lowest}_{\log }$ FC at the top.

Author Contributions: Conceptualization, H.M.E. and X.-Q.Z.; methodology, M.S.R.H.; software, M.S.R.H.; validation, M.S.R.H. and H.M.E.; formal analysis, M.S.R.H. and M.T.; investigation, M.S.R.H., M.T. and H.M.E.; resources, M.S.R.H., X.-Q.Z. and H.M.E.; data curation, M.S.R.H.; writing—original draft preparation, M.S.R.H.; writing-review and editing, X.-Q.Z and H.M.E.; visualization, M.S.R.H. and H.M.E.; supervision, H.M.E.; project administration, H.M.E.; funding acquisition, X.-Q.Z and H.M.E. All authors have read and agreed to the published version of the manuscript.

Funding: This research was supported by a grant from the International Science and Technology Cooperation Project of Gansu Provincial Key Research and Development Program (Grant No. 17JR7WA031) to Xing-Quan Zhu 
and Hany M. Elsheikha. The PhD program of Mohammad S. R. Harun was supported by a scholarship from the Malaysian Ministry of Higher Education.

Acknowledgments: We thank Anton J. Enright for assistance in microarray data analysis.

Conflicts of Interest: The authors declare that they have no conflicts of interest. The funders had no role in the design of the study; in the collection, analyses, or interpretation of data; in the writing of the manuscript, or in the decision to publish the results.

\section{References}

1. Montoya, J.; Liesenfeld, O. Toxoplasmosis. Lancet 2004, 363, 1965-1976. [CrossRef]

2. Elsheikha, H.M. Congenital toxoplasmosis: Priorities for further health promotion action. Public Health 2008, 122, 335-353. [CrossRef] [PubMed]

3. Tenter, A.M.; Heckeroth, A.R.; Weiss, L.M. Toxoplasma gondii: From animals to humans. Int. J. Parasitol. 2000, 30, 1217-1258. [CrossRef]

4. Elsheikha, H.M.; Büsselberg, D.; Zhu, X.Q. The known and missing links between Toxoplasma gondii and schizophrenia. Metab. Brain Dis. 2016, 31, 749-759. [CrossRef] [PubMed]

5. Elsheikha, H.M.; Zhu, X.Q. Toxoplasma gondii infection and schizophrenia: An inter-kingdom communication perspective. Curr. Opin. Infect. Dis. 2016, 29, 311-318. [CrossRef] [PubMed]

6. Sutterland, A.L.; Fond, G.; Kuin, A.; Koeter, M.W.; Lutter, R.; Van Gool, T.; Yolken, R.; Szoke, A.; Leboyer, M.; De Haan, L. Beyond the association. Toxoplasma gondii in schizophrenia, bipolar disorder, and addiction: Systematic review and meta-analysis. Acta Psychiatr. Scand. 2015, 132, 161-179. [CrossRef]

7. Leport, C.; Raddi, F.; Metherton, S.; Katlama, C.; Regnier, B.; Simot, A.G.; Marche, C.; Verdenne, C.; Vilde, J.L. Treatment of central nervous system toxoplasmosis with pyrimethamine/sulfadiazine combination in 35 patients with the acquired immunodeficiency syndrome. Am. J. Med. 1988, 84, 94-100. [CrossRef]

8. Schmidt, D.R.; Hogh, B.; Andersen, O.; Hansen, S.H.; Dalhoff, K.; Petersen, E. Treatment of infants with congenital toxoplasmosis: Tolerability and plasma concentrations of sulfadiazine and pyrimethamine. Eur. J. Pediatr. 2006, 165, 19-25. [CrossRef]

9. Feustel, S.M.; Meissner, M.; Liesenfeld, O. Toxoplasma gondii and the blood-brain barrier. Virulence 2012, 3 , 182-192. [CrossRef]

10. Jia, B.; Lu, H.; Liu, Q.; Yin, J.; Jiang, N.; Chen, Q. Genome-wide comparative analysis revealed significant transcriptome changes in mice after Toxoplasma gondii infection. Parasit. Vectors 2013, 6, 161. [CrossRef]

11. Kessler, M.; Zielecki, J.; Thieck, O.; Mollenkopf, H.J.; Fotopoulou, C.; Meyer, T.F. Chlamydia trachomatis disturbs epithelial tissue homeostasis in fallopian tubes via paracrine Wnt signaling. Am. J. Pathol. 2012, 180, 186-198. [CrossRef] [PubMed]

12. Shapira, S.D.; Gat-Viks, I.; Shum, B.O.V.; Dricot, A.; De Grace, M.M.; Wu, L.; Gupta, P.B.; Hao, T.; Silver, S.J.; Root, D.E.; et al. A physical and regulatory map of host-influenza interactions reveals pathways in H1N1 infection. Cell 2009, 139, 1255-1267. [CrossRef] [PubMed]

13. Clevers, H.; Nusse, R. Wnt/ $\beta$-catenin signaling and disease. Cell 2012, 149, 1192-1205. [CrossRef] [PubMed]

14. Kapoor, A.; He, R.; Venkatadri, R.; Forman, M.; Arav-Boger, R. Wnt modulating agents inhibit human Cytomegalovirus replication. Antimicrob. Agents Chemother. 2013, 57, 2761-2767. [CrossRef] [PubMed]

15. Rutkowski, J.; Brzezinski, B. Structures and properties of naturally occurring polyether antibiotics. Biomed Res. Int. 2013, 2013, 162513. [CrossRef]

16. Callaway, T.; Edrington, T.; Rychlik, J. Ionophores: Their use as ruminant growth promotants and impact on food safety. Curr. Issues Intest. Microbiol. 2003, 4, 43-51.

17. Teichmann, K.; Kuliberda, M.; Schatzmayr, G.; Hadacek, F.; Joachim, A. In vitro determination of anticryptosporidial activity of phytogenic extracts and compounds. Parasitol. Res. 2012, 111, 231-240. [CrossRef]

18. Kim, S.H.; Kim, K.Y.; Yu, S.N.; Park, S.G.; Yu, H.S.; Seo, Y.K.; Ahn, S.C. Monensin induces PC-3 prostate cancer cell apoptosis via ROS production and $\mathrm{Ca}^{2+}$ homeostasis disruption. Anticancer Res. 2016, 36, 5835-5843. [CrossRef]

19. Deng, Y.; Zhang, J.; Wang, Z.; Yan, Z.; Qiao, M.; Ye, J.; Wei, Q.; Wang, J.; Wang, X.; Zhao, L.; et al. Antibiotic monensin synergizes with EGFR inhibitors and oxaliplatin to suppress the proliferation of human ovarian cancer cells. Sci. Rep. 2015, 5, 17523. [CrossRef] 
20. Vichai, V.; Kirtikara, K. Sulforhodamine B colorimetric assay for cytotoxicity screening. Nat. Protoc. 2006, 1, 1112-1116. [CrossRef]

21. Irizarry, R.A.; Bolstad, B.M.; Collin, F.; Cope, L.M.; Hobbs, B.; Speed, T.P. Summaries of Affymetrix GeneChip probe level data. Nucleic Acids Res. 2003, 31, e15. [CrossRef] [PubMed]

22. Irizarry, R.A.; Hobbs, B.; Collin, F.; Beazer-Barclay, Y.D.; Antonellis, K.J.; Scherf, U.; Speed, T.P. Exploration, normalization, and summaries of high density oligonucleotide array probe level data. Biostatistics 2003, 4, 249-264. [CrossRef] [PubMed]

23. Ashburner, M.; Ball, C.A.; Blake, J.A.; Botstein, D.; Butler, H.; Cherry, J.M.; Davis, A.P.; Dolinski, K.; Dwight, S.S.; Eppig, J.T.; et al. Gene ontology: Tool for the unification of biology. The Gene Ontology Consortium. Nat. Genet. 2000, 25, 25-29. [CrossRef] [PubMed]

24. Mi, H.; Muruganujan, A.; Casagrande, J.T.; Thomas, P.D. Large-scale gene function analysis with the panther classification system. Nat. Protoc. 2013, 8, 1551-1566. [CrossRef] [PubMed]

25. Mi, H.; Huang, X.; Muruganujan, A.; Tang, H.; Mills, C.; Kang, D.; Thomas, P.D. PANTHER Version 11: Expanded annotation data from Gene Ontology and Reactome pathways, and data analysis tool enhancements. Nucleic Acids Res. 2017, 45, D183-D189. [CrossRef]

26. Lavine, M.D.; Arrizabalaga, G. The antibiotic monensin causes cell cycle disruption of Toxoplasma gondii mediated through the DNA repair enzyme TgMSH-1. Antimicrob. Agents Chemother. 2011, 55, 745-755. [CrossRef]

27. Lavine, M.D.; Arrizabalaga, G. Analysis of monensin sensitivity in Toxoplasma gondii reveals autophagy as a mechanism for drug induced death. PLoS ONE 2012, 7, e42107. [CrossRef]

28. Barragan, A.; Sibley, L.D. Migration of Toxoplasma gondii across biological barriers. Trends Microbiol. 2003, 11, 426-430. [CrossRef]

29. Dalton, J.E.; Cruickshank, S.M.; Egan, C.E.; Mears, R.; Newton, D.J.; Andrew, E.M.; Lawrence, B.; Howell, G.; Else, K.J.; Gubbels, M.-J.; et al. Intraepithelial gammadelta+ lymphocytes maintain the integrity of intestinal epithelial tight junctions in response to infection. Gastroenterology 2006, 131, 818-829. [CrossRef]

30. Weidner, J.M.; Kanatani, S.; Hernández-Castañeda, M.A.; Fuks, J.M.; Rethi, B.; Wallin, R.P.A.; Barragan, A. Rapid cytoskeleton remodelling in dendritic cells following invasion by Toxoplasma gondii coincides with the onset of a hypermigratory phenotype. Cell. Microbiol. 2013, 15, 1735-1752. [CrossRef]

31. De Vries, H.E.; Kuiper, J.; De Boer, A.G.; Van Berkel, T.J.; Breimer, D.D. The blood-brain barrier in neuroinflammatory diseases. Pharmacol. Rev. 1997, 49, 143-155. [PubMed]

32. Elsheikha, H.M.; Khan, N.A. Protozoa traversal of the blood-brain barrier to invade the central nervous system. FEMS Microbiol. Rev. 2010, 34, 532-553. [CrossRef] [PubMed]

33. Lambert, H.; Vutova, P.P.; Adams, W.C.; Loré, K.; Barragan, A. The Toxoplasma gondii-shuttling function of dendritic cells is linked to the parasite genotype. Infect. Immun. 2009, 77, 1679-1688. [CrossRef]

34. Couzinet, S.; Dubremetz, J.F.; Buzoni-Gatel, D.; Jeminet, G.; Prensier, G. In vitro activity of the polyether ionophorous antibiotic monensin against the cyst form of Toxoplasma gondii. Parasitology 2000, 121, 359-365. [CrossRef] [PubMed]

35. Wang, Z.; Suo, X.; Xia, X.; Shen, J. Influence of monensin on cation influx and Na+ -K+ -ATPase activity of Eimeria tenella sporozoites in vitro. J. Parasitol. 2006, 92, 1092-1096. [CrossRef] [PubMed]

36. Tartakoff, A.M. Perturbation of vesicular traffic with the carboxylic ionophore monensin. Cell 1983, 32, 1026-1028. [CrossRef]

37. Badger, J.L.; Stins, M.F.; Kim, K.S. Citrobacter freundii invades and replicates in human brain microvascular endothelial cells. Infect. Immun. 1999, 67, 4208-4215. [CrossRef]

38. Miraglia, M.C.; Rodriguez, A.M.; Barrionuevo, P.; Rodriguez, J.; Kim, K.S.; Dennis, V.A.; Delpino, M.V.; Giambartolomei, G.H. Brucella abortus traverses brain microvascular endothelial cells using infected monocytes as a Trojan horse. Front. Cell. Infect. Microbiol. 2018, 8, 200. [CrossRef]

39. Wang, X.; Wu, X.; Zhang, Z.; Ma, C.; Wu, T.; Tang, S.; Zeng, Z.; Huang, S.; Gong, C.; Yuan, C.; et al. Monensin inhibits cell proliferation and tumor growth of chemo-resistant pancreatic cancer cells by targeting the EGFR signaling pathway. Sci. Rep. 2018, 8, 17914. [CrossRef]

40. Tumova, L.; Pombinho, A.R.; Vojtechova, M.; Stancikova, J.; Gradl, D.; Krausova, M.; Sloncova, E.; Horazna, M.; Kriz, V.; Machonova, O.; et al. Monensin inhibits canonical Wnt signaling in human colorectal cancer cells and suppresses tumor growth in multiple intestinal neoplasia mice. Mol. Cancer Ther. 2014, 13, 812-822. [CrossRef] 
41. Zhai, B.; He, J.; Elsheikha, H.M.; Li, J.X.; Zhu, X.Q.; Yang, X. Transcriptional changes in Toxoplasma gondii in response to treatment with monensin. Parasit. Vectors 2020, 13, 84. [CrossRef] [PubMed]

42. Melnik, S.; Dvornikov, D.; Müller-Decker, K.; Depner, S.; Stannek, P.; Meister, M.; Warth, A.; Thomas, M.; Muley, T.; Risch, A.; et al. Cancer cell specific inhibition of Wnt/B-Catenin signaling by forced intracellular acidification. Cell Discov. 2018, 4, 37. [CrossRef] [PubMed]

43. Ketola, K.; Vainio, P.; Fey, V.; Kallioniemi, O.; Iljin, K. Monensin is a potent inducer of oxidative stress and inhibitor of androgen signaling leading to apoptosis in prostate cancer cells. Mol. Cancer Ther. 2010, 9, 3175-3185. [CrossRef] [PubMed]

44. Oh, I.; Reddy, E. The Myb gene family in cell growth, differentiation and apoptosis. Oncogene 1999, 18, 3017-3033. [CrossRef] [PubMed]

45. Ramsay, R.; Gonda, T. MYB function in normal and cancer cells. Nat. Rev. Cancer 2008, 8, 523-534. [CrossRef] [PubMed]

46. Yusenko, M.; Trentmann, A.; Andersson, M.; Ghani, L.; Jakobs, A.; Arteaga Paz, M.; Mikesch, J.; Peter von Kries, J.; Stenman, G.; Klempnauer, K. Monensin, a novel potent MYB inhibitor, suppresses proliferation of acute myeloid leukemia and adenoid cystic carcinoma cells. Cancer Lett. 2020, 479, 61-70. [CrossRef]

47. Ngô, H.M.; Zhou, Y.; Lorenzi, H.; Wang, K.; Kim, T.K.; Zhou, Y.; Bissati, K.E.; Mui, E.; Fraczek, L.; Rajagopala, S.V.; et al. Toxoplasma modulates signature pathways of human epilepsy, neurodegeneration \& cancer. Sci. Rep. 2017, 7, 11496. [CrossRef]

48. Zhang, X.; Su, R.; Cheng, Z.; Zhu, W.; Li, Y.; Wang, Y.; Du, J.; Cai, Y.; Luo, Q.; Shen, J.; et al. A mechanistic Study of Toxoplasma gondii ROP18 inhibiting differentiation of C17.2 neural stem cells. Parasit. Vectors 2017, 10, 585. [CrossRef]

49. Lüder, C.G.K.; Lang, C.; Giraldo-Velasquez, M.; Algner, M.; Gerdes, J.; Gross, U. Toxoplasma gondii inhibits MHC class II expression in neural antigen-presenting cells by down-regulating the class II transactivator CIITA. J. Neuroimmunol. 2003, 134, 12-24. [CrossRef]

50. Kim, S.K.; Fouts, A.E.; Boothroyd, J.C. Toxoplasma gondii dysregulates IFN-gamma-inducible gene expression in human fibroblasts: Insights from a genome-wide transcriptional profiling. J. Immunol. 2007, 178, 5154-5165. [CrossRef]

51. Al-Sandaqchi, A.T.; Brignell, C.; Collingwood, J.F.; Geraki, K.; Mirkes, E.M.; Kong, K.; Castellanos, M.; May, S.T.; Stevenson, C.W.; Elsheikha, H.M. Metallome of cerebrovascular endothelial cells infected with Toxoplasma gondii using $\mu$-XRF imaging and inductively coupled plasma mass spectrometry. Metallomics 2018, 10, 1401-1414. [CrossRef] [PubMed]

52. Cannella, D.; Brenier-Pinchart, M.P.; Braun, L.; Van Rooyen, J.M.; Bougdour, A.; Bastien, O.; Behnke, M.S.; Curt, R.L.; Curt, A.; Saeij, J.P.; et al. miR-146a and miR-155 delineate a MicroRNA fingerprint associated with Toxoplasma persistence in the host brain. Cell Rep. 2014, 6, 928-937. [CrossRef] [PubMed] 